\title{
On Invariants $G(\sigma)$ and $\Gamma(\sigma)$ for an Automorphism Group of a von Neumann Algebra
}

\author{
$\mathrm{By}$
}

\author{
Akio IKUNISHI* and Yoshiomi NAKAGAMI**
}

\begin{abstract}
An invariant $\Gamma$ for an automorphism group of a factor given by Connes is generalized to a general von Neumann algebra and the relation between $\Gamma$ and a characterization of an inner automorphism group of a von Neumann algebra due to Borchers are discussed.
\end{abstract}

\section{§ 1. Introduction}

Let $G$ be a locally compact abelian group, $d t$ a Haar measure on $G, \widehat{G}$ the dual of $G$ and $\langle t, \gamma\rangle$ the value of $\gamma \in \widehat{G}$ at $t \in G$. For $g \in L^{1}(G)$ and $\gamma \in \widehat{G}$

$$
\widehat{g}(\gamma) \equiv \int_{G} g(t) \overline{\langle t, \gamma\rangle} d t
$$

and $\Gamma(g) \equiv\{\gamma \in \widehat{G}: \widehat{g}(\gamma)=0\}$.

Let $M$ be a von Neumann algebra, $M_{*}$ the predual of $M$ and Aut $M$ the group of automorphisms of $M$. A homomorphism $\sigma$ of $G$ into Aut $M$ satisfying that the functions $t \in G \mapsto \phi\left(\sigma_{t}(x)\right)$ are continuous for all $x \in M$ and $\phi \in M_{*}$ is called a representation of $G$ on $M$. Let $\operatorname{Rep}(G, M)$ denote the set of all representations of $G$ on $M$. For a finite measure $\mu$ on $G$ (resp. $\left.g \in L^{1}(G)\right), \sigma \in \operatorname{Rep}(G, M)$ and $x \in M$ let

$$
\sigma(\mu) x \equiv \int_{G} \sigma_{t}(x) \mu(d t) \quad\left(\operatorname{resp} . \sigma(g) x \equiv \int_{G} g(t) \sigma_{t}(x) d t\right) .
$$

Let $\operatorname{sp} \sigma$ denote the intersection of $\Gamma(g)$ with $\sigma(g)=0$ and $\operatorname{sp}_{\sigma}(x)$ the intersection of $\Gamma(g)$ with $\sigma(g) x=0$. For a closed subset $E$ of $\widehat{G}, M^{\sigma}(E)$

Communicated by H. Araki, August 23, 1974.

* Department of Applied Physics, Tokyo Institute of Technology, Tokyo.

** Department of Mathematics, Tokyo Institute of Technology, Tokyo.

Present address: Department of Mathematics, Kyushu University, Fukuoka. 
denotes the set of all $x \in M$ with $\operatorname{sp}_{\sigma}(x) \subset E$. Let $M^{\sigma} \equiv M^{\sigma}(\{0\}), Z(M)$ $\equiv M \cap M^{\prime}$ and $Z\left(M^{\sigma}\right) \equiv M^{\sigma} \cap\left(M^{\sigma}\right)^{\prime}$. For projections $e$ and $f$ in $M^{\sigma}, \bar{e}$ denotes the carrier in $Z(M)$ of $e$ and ${ }^{e} \sigma^{f}$ the restriction defined by

$$
{ }^{e} \sigma_{t}^{f}(x) \equiv \sigma_{t}(x), \quad x \in e M f,
$$

in particular, $\sigma^{e} \equiv{ }^{e} \sigma^{e}$ or $\sigma^{e}$ is the restriction of $\sigma$ to $M_{e}$. Furthermore, $\operatorname{sp}^{e} \sigma^{f}$ denotes the intersection of $\Gamma(g)$ with ${ }^{e} \sigma^{f}(g)=0$, where ${ }^{e} \sigma^{f}(g)$ is defined similarly as above.

Definition 1.1. $G(\sigma)$ (resp. $K(\sigma)$ ) denotes the set of all $t \in G$ such that $\sigma_{t}$ is implemented by a unitary in $M^{\sigma}(\operatorname{resp} . M)$.

Then $G(\sigma)$ and $K(\sigma)$ are subgroups of $G$ and $G(\sigma) \subset K(\sigma)$. The following definition is essentially due to Connes, [6].

Definition 1.2. $\Gamma_{0}(\sigma)$ (resp. $\Gamma_{1}(\sigma)$ ) denotes the intersection of all $\operatorname{sp} \sigma^{e}, e \in M^{\sigma}$ with $e \neq 0$ (resp. $\bar{e}=1$ ).

Then $\Gamma_{0}(\sigma)$ is a closed subgroup of $\widehat{G}$ and $\Gamma_{0}(\sigma) \subset \Gamma_{1}(\sigma)$. There is no difference between $\Gamma_{0}(\sigma)$ and $\Gamma_{1}(\sigma)$ if $M$ is a factor.

The main purpose of this paper is to show the relations among the following four conditions for a closed subgroup $\Xi$ of $\widehat{G}$ :

(A) for any non zero projection $f$ in $Z\left(M^{\sigma}\right)$ and for any neighbourhood $V$ of 0 there exists a non zero projection $e$ in $Z\left(M^{\sigma}\right)$ such that $e \leqq f$ and $\Xi \subset \operatorname{sp} \sigma^{e} \subset \Xi+V$;

(B) $G\left(\sigma^{e}\right)=\Xi^{\perp}$ for all non zero $e$ in $Z\left(M^{\sigma}\right)$;

(B') $G\left(\sigma^{e}\right)=\Xi^{\perp}$ and $\Xi \subset \operatorname{sp} \sigma^{e}$ for all non zero $e$ in $Z\left(M^{\sigma}\right)$;

(C) $\Gamma_{0}(\sigma)=\Gamma_{1}(\sigma)=\Xi$.

Using these conditions, we can state our main theorem.

Theorem 1.1. (i) The condition (A) implies the condition ( $\left.\mathrm{B}^{\prime}\right)$, and the condition $\left(\mathrm{B}^{\prime}\right)$ implies the condition (C).

(ii) If $\widehat{G} / \Xi$ is compact, then conditions $(\mathrm{A}),\left(\mathrm{B}^{\prime}\right)$ and $(\mathrm{C})$ are equivalent.

(iii) If $G$ satisfies the second axiom of countability, $\Xi=\{0\}$ and 
$M_{*}$ is separable, then conditions (A) and (B) are equivalent. In this case, $\sigma$ is inner.

The implications $(A) \Rightarrow(B)$ and $\left(B^{\prime}\right) \Rightarrow(C)$ are proved in Section 2 by similar techniques as Borchers, [4]. The implication (C) $\Rightarrow(A)$ for a discrete $\Xi^{\perp}$ is proved in Section 3 by similar ideas as Connes, [6]. The statement (iii) implies the following corollary.

Corollary 1.1. If $G$ satisfies the second axiom of countability, $G(\sigma)=G$ and $M_{*}$ is separable, then $\sigma$ is inner.

The ergodicity of $\sigma$ implies the equivalence of conditions (A), (B) and (C), whenever $\widehat{G} / \Xi$ is compact (Remark 3.2 ).

Let $W(M)$ be the set of semi-finite, faithful and normal weights on $M_{+}$. For $\phi \in W(M), \Delta_{\phi}$ and $\sigma^{\phi}$ denote the modular operator and modular automorphism of $\phi$, respectively.

Definition 1.3. $\mathrm{S}(M)$ denotes the intersection of all spectrum of $\Delta_{\phi}$, $\phi \in W(M)$.

Theorem 1.2. If $\Gamma_{0}\left(\sigma^{\phi}\right)=\Gamma_{1}\left(\sigma^{\phi}\right) \neq\{0\}$, then $\log \left(\mathrm{S}\left(M_{e}\right) \backslash\{0\}\right)$ $=\Gamma_{0}\left(\sigma^{\phi}\right)$ for any non zero $e$ in $Z(M)$.

Finally, in Section 5 we shall give a characterization of an unbounded derivation which corresponds to a representation of $\boldsymbol{R}$ on $M$.

\section{$\S 2$. Proof of (i) in Theorem 1.1}

The condition (A) implies the existence of a projection $e_{0}$ in $M^{\sigma}$ (or $Z\left(M^{\sigma}\right)$ ) such that $\Xi \subset \mathrm{sp} \sigma^{e_{0}} \subset \Xi+V$ and $\bar{e}_{0}=1$. For this, let $\mathscr{Z}$ be the family of sets of non zero projections $e$ in $M^{\sigma}$ (or $Z\left(M^{\sigma}\right)$ ) such that $\Xi \subset \operatorname{sp} \sigma^{e} \subset \Xi+V$ and their central carriers in $M$ are mutually orthogonal. Then $\mathscr{Z}$ is a non empty ordered set by set inclusion. Here we apply Zorn's lemma to $\mathscr{F}$ and obtain a maximal set $\left\{\boldsymbol{e}_{\alpha}\right\} \in \mathscr{F}$. We complete the proof by defining $e_{0}$ by $\sum e_{\alpha}$. Therefore the condition (A) implies that $\Xi \subset \Gamma_{0}(\sigma) \subset \Gamma_{1}(\sigma) \subset \cap\{\Xi+V$ : as above $\}=\Xi$, which implies the condition (C). 
It is known that $K\left(\sigma^{e}\right)=K\left(\sigma^{\bar{e}}\right)$ for $e \in M^{\sigma}$, [4, Lemma 5.7; 6, Lemma 1.5.2].

Lemma 2.1. $G\left(\sigma^{e}\right)=G\left(\sigma^{\bar{e}}\right)$ for $e \in M^{\sigma}$.

Proof. Since $G\left(\sigma^{\bar{e}}\right) \subset G\left(\sigma^{e}\right)$, it suffices to show the converse inclusion for a non zero $e$ in $M^{\sigma}$. Suppose that $t \in G\left(\sigma^{e}\right)$. Then we have a unitary $v$ on $e \mathcal{H}$ such that $v \in M_{e}{ }^{\sigma}$ and $\sigma_{t}{ }^{e}(x)=v x v^{*}$ for $x \in M_{e}$. Define an operator $u$ on $\bar{e} \mathcal{H}$ by $u y \xi \equiv \sigma_{t}^{\bar{e}}(y) v \xi$ for $y \in M_{\bar{e}}$ and $\xi \in e \mathcal{H}$. Since

$$
(u y \xi \mid u z \eta)=\left(\sigma_{t}^{e}\left(z^{*} y\right) v \xi \mid v \eta\right)=(y \xi \mid z \eta)
$$

whenever $\xi, \eta \in e \mathcal{H}, u$ is a unitary in $M_{\bar{e}}$ such that $u_{e}=v$ and $\sigma_{t}^{\bar{e}}(y)$ $=u y u^{*}$ for $y \in M_{\bar{e}}$. Since $v \in M^{\sigma} \cap M_{e}$, we have $e \sigma_{s}^{\bar{e}}(u)=\sigma_{s}^{\bar{e}}(u) e$ and $\left(\sigma_{s}^{\bar{e}}(u)\right)_{e}=v$ for all $s \in G$. For $s \in G, y \in M_{\bar{e}}$ and $\xi \in e \mathcal{H}$, we have

$$
\begin{aligned}
y v \xi & =y \sigma_{s}^{\bar{e}}(u) \xi=\sigma_{s}^{\bar{e}}\left(\sigma_{-s}^{\bar{e}}(y) u\right) \xi \\
& =\sigma_{s}^{\bar{e}}\left(u \sigma_{-t-s}^{\bar{e}}(y)\right) \xi=\sigma_{s}^{\bar{e}}(u) \sigma_{-t}^{\bar{e}}(y) \xi=\sigma_{s}^{\bar{e}}(u) u^{*} y v \xi
\end{aligned}
$$

Therefore $\sigma_{s}^{\bar{e}}(u)=u$ for all $s \in G$ and hence $u \in M^{\sigma} \cap M_{\bar{e}}$. Consequently we have $G\left(\sigma^{e}\right) \subset G\left(\sigma^{\bar{e}}\right)$.

Q.E.D.

This lemma implies the equivalence between the conditions (B) and

$\left(\mathrm{B}_{1}\right) G\left(\sigma^{f}\right)=\Xi^{\perp}$ for all non zero $f$ in $Z(M) \cap Z\left(M^{\sigma}\right)$.

Let $\tau$ be a representation of $Z$ defined by $\tau_{n}=\sigma_{n t}$ for some fixed $t \in G$ in this section. $\left\langle t, \operatorname{sp}_{\sigma}(x)\right\rangle$ denotes the set of all $\langle t, \gamma\rangle$ with $\gamma \in$ $\operatorname{sp}_{\sigma}(x)$. In the following lemmas we shall identify the dual of $\boldsymbol{Z}$ with the unit circle $\boldsymbol{T}$.

Lemma 2.2. $\left\langle t, \operatorname{sp}_{\sigma}(x)\right\rangle^{-}=\operatorname{sp}_{\tau}(x)$ for all $x \in M$.

Proof. Suppose that $\gamma \in \operatorname{sp}_{\sigma}(x)$. Let $g \in l^{1}(\boldsymbol{Z})$ with $\tau(g) x=0$. By setting $\mu \equiv \sum_{n \in \boldsymbol{Z}} g(n) \delta_{n t}$, we have $\sigma(\mu) x=\tau(g) x=0$ and hence

$$
\widehat{g}(\langle t, \gamma\rangle)=\hat{\mu}(\gamma)=0 .
$$

Therefore we have $\langle t, \gamma\rangle \in \operatorname{sp}_{\tau}(x)$.

Choose any $g \in l^{1}(\boldsymbol{Z})$ so that $\widehat{g}$ vanishes on a neighbourhood $V$ of $\left\langle t, \operatorname{sp}_{\sigma}(x)\right\rangle^{-}$. Setting $\mu \equiv \sum_{n} g(n) \delta_{n t}$, we have 


$$
\hat{\mu}(\gamma)=\widehat{g}(\langle t, \gamma\rangle)=0
$$

on the neighbourhood $\{\gamma \in \widehat{G}:\langle t, \gamma\rangle \in V\}$ of $\operatorname{sp}_{\sigma}(x)$, and so $\tau(g) x=\sigma(\mu) x$ $=0$. Consequently, we have

$$
\operatorname{sp}_{\tau}(x) \subset\left\langle t, \operatorname{sp}_{\sigma}(x)\right\rangle^{-} .
$$

We shall also identify the dual of $Z$ with $(-\pi, \pi]$ and denote $[-\delta, \delta]$ by $\mathrm{I}_{\delta}$. For a projection $e \in Z\left(M^{\tau}\right)$ and a closed subset $E$ of $(-\pi, \pi]$, let $\phi(E, e)$ denote the projection onto the closed subspace spanned by $M^{r}(E) e \mathcal{H}$. Since Lemma 2.2 implies

$$
M^{\sigma}(\{\gamma \in \widehat{G}:\langle t, \gamma\rangle \in E\})=M^{*}(E),
$$

we have $\phi(E, e) \in Z\left(M^{\sigma}\right)$ for $e \in Z\left(M^{\sigma}\right)$.

The following Lemmas 2.3 and 2.4 have been obtained by Borchers, [4], while we shall give their proofs for completeness.

Assume that $\langle t, \operatorname{sp} \sigma\rangle^{-} \subset(-2 \pi / 3,2 \pi / 3)$ in Lemmas 2.3, 2.4 and 2.5.

Lemma 2.3. There exists a family $\{p(\delta): \delta \in(0,2 \pi / 3)\}$ of projections in $Z\left(M^{\sigma}\right)$ such that $p(\delta)$ is increasing in $\delta$, $\mathrm{sp} \tau^{p^{(\delta)}} \subset I_{\delta}$ and $\overline{p(\delta)}=1$.

Proof. We shall define $p_{n}$ by induction. Put $\delta_{n} \equiv 2^{-n+2} \pi / 3$ and $p_{1}$ $\equiv 1$. Then $\operatorname{sp} \tau^{\rho_{1}} \subset(-2 \pi / 3,2 \pi / 3)$ by assumption. Assume that $p_{j} \in Z$ $\left(M^{\sigma}\right), p_{j-1} \geq p_{j}$, sp $\tau^{p_{j}} \subset I_{\delta_{j}}$ and $\bar{p}_{j}=1$ for $j=2,3, \cdots, n$. Put

$$
\begin{aligned}
& \delta \equiv 2^{-1} \inf \left\{\varepsilon>0: \operatorname{sp} \tau^{p_{n}} \subset I_{\varepsilon}\right\} \quad(<\pi / 3), \\
& p \equiv p_{n} \phi\left([\delta, 2 \delta+\varepsilon], p_{n}\right) \in Z\left(M^{\sigma}\right), \\
& p_{n+1} \equiv p+p_{n}(1-\bar{p}) \in Z\left(M^{\sigma}\right)
\end{aligned}
$$

for some $\varepsilon \in(0,2 \pi-6 \delta)$. Then $\delta \leq \delta_{n+1}, p_{n+1} \leq p_{n}$ and $\overline{p_{n+1}}=1$. Since $p_{n}$ $(1-\bar{p}) \phi\left([\delta, 2 \delta+\varepsilon], p_{n}\right)=0$, we have

$$
\operatorname{sp}^{p_{n}(1-\tilde{p})} \tau^{p_{n}} \cap(\delta, 2 \delta+\varepsilon)=\emptyset .
$$

Since $\operatorname{sp} \tau^{q} \subset \operatorname{sp}^{q} \tau^{p_{n}}$ and $\operatorname{sp} \tau^{q}=-\operatorname{sp} \tau^{q}$ for $q \equiv p_{n}(1-\bar{p})$,

$$
\operatorname{sp} \tau^{p_{n}(1-\bar{p})} \subset \operatorname{sp} \tau^{p_{n}} \backslash\{(-2 \delta-\varepsilon,-\delta) \cup(\delta, 2 \delta+\varepsilon)\} \subset I_{\delta} .
$$

Since $\operatorname{sp}^{f} \tau^{\phi(E, e)} \subset \operatorname{sp}^{f} \tau^{e}-E$ for $e, f \in Z\left(M^{r}\right)$ in general, 


$$
\begin{aligned}
\operatorname{sp} \tau^{p} & \subset \operatorname{sp}^{p_{n}} \tau^{\phi\left([\delta, 2 \delta+\varepsilon], p_{n}\right)} \cap \operatorname{sp}^{\phi\left([\delta, 2 \delta+\varepsilon], p_{n}\right)} \tau^{p_{n}} \\
& \subset\left(I_{2 \delta}-[\delta, 2 \delta+\varepsilon]\right) \cap\left(I_{2 \delta}+[\delta, 2 \delta+\varepsilon]\right) \subset I_{\delta} .
\end{aligned}
$$

Consequently, we have

$$
\operatorname{sp} \tau^{p_{n+1}}=\operatorname{sp} \tau^{p} \bigcup \mathrm{sp} \tau^{p_{n}^{(1-\bar{p})} \subset I_{\delta} \subset I_{\delta_{n+1}}} .
$$

Putting $p(\delta) \equiv p_{n+1}$ for $\delta \in\left[\delta_{n+1}, \delta_{n}\right), n \in N$, we have a family $\{p(\delta)$ : $\delta \in(0,2 \pi / 3)\}$ with the desired property.

Q.E.D.

Lemma 2.4. For any projection e in $Z\left(M^{\sigma}\right)$, put

$$
S(e) \equiv \bigcap\left\{\mathrm{sp}^{e} \tau^{p(\delta)}: \delta>0\right\} .
$$

Then for any $e_{1}, e_{2}, e_{\alpha}, e, f$ in $Z\left(M^{\sigma}\right)$ and any closed subset $E$ of $(-\pi, \pi]$, it holds that
(a) $S\left(e_{1}\right) \subset S\left(e_{2}\right)$ if $e_{1} \leq e_{2}$;
(b) $S(p(\delta)) \subset I_{\delta}$;
(c) $S(\phi(E, e)) \subset S(e)+E$;
(d) $S(e)=\emptyset$ if and only if $e=0$;
(e) $\quad\left(\cup S\left(e_{\alpha}\right)\right)^{-}=S\left(\sup e_{\alpha}\right)$;
(f) $\operatorname{sp}^{e} \tau^{f} \subset S(e)-S(f)$; and
(g) $e M^{r}(E) f \subset M^{r}(\{S(e)-S(f)\} \cap E)$.

Proof. (a) and (b) are obvious.

(c) We have

$$
S(\phi(E, e)) \equiv \bigcap_{\delta>0} \operatorname{sp}^{\phi(E, e)} \tau^{p(\delta)} \subset \bigcap_{\delta>0}\left(\operatorname{sp}^{e} \tau^{p(\delta)}+E\right) .
$$

Since $E$ is compact, it follows that

$$
\bigcap_{\delta>0}\left(\operatorname{sp}^{e} \tau^{p(\delta)}+E\right)=\bigcap_{\delta>0} \operatorname{sp}^{e} \tau^{p(\delta)}+E \equiv S(e)+E .
$$

(d) $e=0$ clearly implies $S(e)=\emptyset . \quad B y$ compactness, $S(e)=\emptyset$ implies $\operatorname{sp}^{e} \tau^{p(\delta)}=\emptyset$ for some $\delta>0$. Therefore $e M p(\delta)=\{0\}$. Since $\overline{p(\delta)}$ $=1, e=0$.

(e) For any $\delta>0$ and $\varepsilon \in(0, \delta)$, since $\operatorname{sp} \tau^{p(\delta)} \subset I_{\delta}, p(\delta) \phi\left(I_{\delta}, p(\varepsilon)\right)$ is the carrier in $Z\left(M_{p(\delta)}\right)$ of $p(\varepsilon)$ and hence $p(\delta) \leq \phi\left(I_{\delta}, p(\varepsilon)\right)$. Therefore

$$
\operatorname{sp}^{e} \tau^{p(\delta)} \subset \bigcap_{\varepsilon>0} \operatorname{sp}^{e} \tau^{\phi\left(I_{\delta}, p(\varepsilon)\right)} \subset S(e)+I_{\delta}
$$


Therefore

$$
\begin{aligned}
& S\left(\sup e_{\alpha}\right)=\bigcap_{\delta>0}\left(\bigcup_{\alpha} \operatorname{sp}^{e_{\alpha}} \tau^{p(\delta)}\right)^{-} \subset \bigcap_{\delta>0}\left(\bigcup_{\alpha}\left(S\left(e_{\alpha}\right)+I_{\delta}\right)\right)^{-} \\
& \subset \bigcap_{\delta>0}\left(\bigcup_{\alpha} S\left(e_{\alpha}\right)+I_{\delta}\right)^{-}=\bigcap_{\delta>0}\left\{\left(\bigcup_{\alpha} S\left(e_{\alpha}\right)\right)^{-}+I_{\delta}\right\}=\left(\bigcup_{\alpha} S\left(e_{\alpha}\right)\right)^{-} .
\end{aligned}
$$

The converse inclusion is clear from (a).

(f) and (g) From (2.1) it follows that $e M p(\delta) \subset M^{\mathfrak{r}}\left(S(e)+I_{\delta}\right)$ and $p(\delta) M f \subset M^{r}\left(-S(f)+I_{\hat{\delta}}\right)$. Therefore

$$
\begin{gathered}
e M p(\delta) M f \subset M^{\tau}\left(S(e)+I_{\delta}\right) M^{\tau}\left(-S(f)+I_{\delta}\right) \\
\subset M^{\tau}\left(S(e)-S(f)+I_{2 \delta}\right) .
\end{gathered}
$$

Since $\overline{p(\delta)}=1, M p(\delta) M$ is weakly total in $M$. Therefore

$$
e M f \subset M^{\tau}\left(S(e)-S(f)+I_{2 \delta}\right),
$$

and hence

$$
\operatorname{sp}^{e} \tau^{f} \subset S(e)-S(f)+I_{2 \delta} .
$$

By the arbitrariness of $\delta>0$, we have (f) and

$$
\begin{gathered}
e M^{r}(E) f \subset M^{r}(S(e)-S(f)) \cap M^{r}(E) \\
\quad=M^{r}(\{S(e)-S(f)\} \cap E),
\end{gathered}
$$

which is $(g)$.

Q.E.D.

\section{Lemma 2.5. Let}

$$
e(\lambda) \equiv \sup \left\{e \in Z\left(M^{\sigma}\right): S(e) \subset(-\pi, \lambda]\right\} .
$$

Then $\{e(\lambda): \lambda \in(-\pi, \pi]\}$ is a spectral resolution of the identity which satisfies

(h) $S(e(\lambda, \mu]) \subset[\lambda, \mu]$.

Proof. It is clear that $e(\lambda)$ is increasing in $\lambda$. Since $S(e(\lambda))$ $\subset(-\pi, \lambda]$ by (e), we have

$$
S\left(\lim _{\mu \downarrow \lambda} e(\mu)\right) \subset \bigcap_{\mu>\lambda} S(e(\mu)) \subset \bigcap_{\mu>\lambda}(-\pi, \mu]=(-\pi, \lambda],
$$

and hence $\lim _{\mu \downarrow \lambda} e(\mu) \leq e(\lambda)$. Therefore $e(\lambda)$ is right continuous in $\lambda$. 
Since $\overline{\langle t, \operatorname{sp} \sigma\rangle} \subset(-\pi, \pi)$ by assumption, it follows from (d) that

$$
\lim _{\lambda \downarrow-\pi} e(\lambda)=0 \quad \text { and } \quad \lim _{\lambda \uparrow \pi} e(\lambda)=1 .
$$

(h) If $\alpha \in(-\pi, \lambda)$, there is a $\delta>0$ with $\alpha+I_{2 \delta} \in(-\pi, \lambda)$, and hence $S\left(\phi\left(\alpha+I_{\delta}, p(\delta)\right)\right) \subset(-\pi, \lambda)$ by (c). It follows that $e((\lambda, \mu]) \phi$ $\left(\alpha+I_{\delta}, p(\delta)\right)=0$ and hence $\alpha \notin \mathrm{sp}^{e(\lambda, \mu]} \tau^{p(\delta)}$. Therefore $\alpha \notin S(e(\lambda, \mu])$.

Q.E.D.

Proof of $(\mathrm{A}) \Rightarrow(\mathrm{B})$. Suppose that $t \in \Xi^{\perp}$. The condition (A) assures the existence of a projection $q \in Z\left(M^{\sigma}\right)$ with $\bar{q}=1$ and $\left\langle t \text {, sp } \sigma^{q}\right\rangle^{-}$ $\subset(-2 \pi / 3,2 \pi / 3)$. For the proof of $\Xi^{\perp} \subset G(\sigma)$ we may assume by Lemma 2.1 that $\langle t, \operatorname{sp} \sigma\rangle^{-} \subset(-2 \pi / 3,2 \pi / 3)$.

Using a spectral resolution $\{e(\lambda): \lambda \in(-\pi, \pi]\}$ obtained in Lemma 2.5 , we define a unitary $u \in Z\left(M^{\sigma}\right)$ and a representation $\rho$ of $\boldsymbol{Z}$ by

$$
u \equiv \int_{-\pi}^{\pi} \exp (-i \lambda) e(d \lambda), \rho_{n} \equiv(\operatorname{Ad} u)^{n}
$$

We shall show that $M^{\mathrm{r}}(E) \subset M^{\rho}(E)$ for any closed $E$. Then, by [2], we have $\rho=\tau$, and so, $t \in G(\sigma)$.

Assume that $\operatorname{sp}_{\tau}(x) \subset E$ and $g \in l^{1}(\boldsymbol{Z})$ such that $\hat{g}$ vanishes on a neighbourhood of $E$. It follows from $(g)$ in Lemma 2.4 and (h) in Lemma 2.5 that

$$
\begin{aligned}
\rho(g) x & =\sum_{n \in \boldsymbol{Z}} g(n) u^{n} x u^{* n} \\
& =\sum_{n} g(n) \iint \exp \{i(\mu-\lambda) n\} e(d \lambda) x e(d \mu) \\
& =\iint \hat{g}(\lambda-\mu) e(d \lambda) x e(d \mu)=0 .
\end{aligned}
$$

Therefore $\operatorname{sp}_{\rho}(x) \subset E$.

Since $\Xi \subset \Gamma_{1}(\sigma)$ by the condition (A), the converse inclusion is clear from the following lemma, which is a partial generalization of [6, Theorem 2.3.1] for a factor.

According to [6, Lemma 2.3.8] we know that the spectrum $\operatorname{Sp}\left(\sigma_{t}\right)$ of $\sigma_{t}$ on $M$ as a Banach space is the closure $\langle t, \operatorname{sp} \sigma\rangle^{-}$of $\{\langle t, \gamma\rangle: \gamma \in \operatorname{sp} \sigma\}$. 
Lemma 2.6. $G(\sigma) \subset \Gamma_{1}(\sigma)^{\perp}$.

Proof. Suppose that $\sigma_{t}(x)=u x u^{*}$ for all $x \in M$ with $u \in M^{\sigma}$. Choose any $\varepsilon>0$. Let $\mathscr{I}$ be the family of sets of $\left(e_{\alpha}, \lambda_{\alpha}\right)$ of spectral projections $e_{\alpha}$ of $u$ and complex numbers $\lambda_{\alpha}$ of modulus 1 such that

(a) $\left\|u e_{\alpha}-\lambda_{\alpha} e_{\alpha}\right\|<\varepsilon$; and

(b) $\bar{e}_{\alpha}$ 's are mutually orthogonal.

Since $\mathscr{F}$ is ordered by set inclusion, we have a maximal set $F \in \mathscr{F}$ by Zorn's lemma, say $F=\left\{\left(e_{\alpha}, \lambda_{\alpha}\right):(\mathrm{a})\right.$, (b) $\}$. By maximality, $\sum \bar{e}_{\alpha}=1$. Let $e \equiv \sum e_{\alpha}$ and $v \equiv \sum \lambda_{\alpha}{ }^{-1} u \bar{e}_{\alpha}$. Then $e \in M^{\sigma}, v$ is a unitary in $M^{\sigma}$ and

$$
\sigma_{t}(x)=u x u^{*}=v x v^{*}
$$

for $x \in M$. Since $\|v e-e\|<\varepsilon$ and

$$
\operatorname{Sp}\left(\sigma_{t}^{e}\right) \subset\left\{\lambda \mu^{-1}: \lambda, \mu \in \operatorname{Sp}\left(v_{e}\right)\right\},
$$

$\operatorname{Sp}\left(\sigma_{t}^{e}\right)$ is included in $\{z \in C:|z|=1,|z-1|<2 \varepsilon\}$. If $\gamma \in \operatorname{sp} \sigma^{e}$, then $\mid<t$, $\gamma\rangle-1 \mid<2 \varepsilon$ by [6, Lemma 2.3.8]. Therefore $|\langle t, \gamma\rangle-1|<2 \varepsilon$ for $\gamma \in \Gamma_{1}(\sigma)$. Since $\varepsilon$ is arbitrary, $t \in \Gamma_{1}(\sigma)^{\perp}$.

Q.E.D.

Remark 2.1. If $\sigma \in$ Aut $M$ satisfies $\|\sigma-1\|<3^{1 / 2}$ and if $G$ is an abelian subgroup of Aut $M$ containing $\sigma$, then there exists a unitary $u \in M$ such that $\sigma=\operatorname{Ad} u$ and $\rho(u)=u$ for all $\rho \in G$.

Remark 2.2. Let $\sigma \in \operatorname{Rep}(G, M)$. Under the condition (A), if $G$ satisfies the first axiom of countability, we can define $S(e), e \in Z\left(M^{\sigma}\right)$ as a subset of $\widehat{G} / \mathrm{G}(\sigma)^{\perp}$ and then $e(\dot{\gamma}) \in Z\left(M^{\sigma}\right), \dot{\gamma} \in \widehat{G} / \mathrm{G}(\sigma)^{\perp}$ as a spectral measure $u_{s}$ :

$$
u_{s} \equiv \int\langle\overline{s, \dot{\gamma}\rangle}\rangle(d \dot{\gamma}), \quad \sigma_{s}=\operatorname{Ad} u_{s}
$$

for all $s \in G(\sigma)$.

Remark 2.3. Let $\sigma \in \operatorname{Rep}(G, M)$. If $G$ is discrete, then $G(\sigma)$ $=\Gamma_{1}(\sigma)^{\perp}$.

Proof of the implication $\left(\mathrm{B}^{\prime}\right) \Rightarrow(\mathrm{C})$. From the condition $(\mathrm{B})$ and 
Lemma 2.6, we have $\Gamma_{1}(\sigma) \subset \Xi$. From the remaining condition of $\left(\mathrm{B}^{\prime}\right)$, we have $\Xi \subset \Gamma_{0}(\sigma)$. Therefore $\Gamma_{0}(\sigma)=\Gamma_{1}(\sigma)=\Xi$.

Q.E.D.

\section{$\S$ 3. Proofs of (ii) and (iii) in Theorem 1.1}

In the following we denote the carrier projection of $x$ by $\mathrm{s}(x)$ and the carrier of $\widehat{g}$ for $g \in L^{1}(G)$ by car $\widehat{g}$.

Lemma 3.1. For any compact neighbourhood $U$ of 0 in $\widehat{G}$ and for any projections $e_{1}$ and $e_{2}$ in $M^{\sigma}$ (resp. $Z\left(M^{\sigma}\right)$ ) with $\bar{e}_{1}=\bar{e}_{2}=1$ there exist projections $f_{1}$ and $f_{2}$ in $M^{\sigma}$ (resp. $\left.Z\left(M^{\sigma}\right)\right)$ such that $\bar{f}_{1}=\bar{f}_{2}=1$, $f_{1} \leq e_{1}, f_{2} \leq e_{2}, \operatorname{sp} \sigma^{f_{1}} \subset U+\operatorname{sp} \sigma^{f_{2}}$ and $\operatorname{sp} \sigma^{f_{2}} \subset U+\operatorname{sp} \sigma^{f_{1}}$.

Proof. Since $\bar{e}_{1}=\bar{e}_{2}=1$, there exists a non zero $x_{0} \in M$ such that $x_{0}=e_{1} x_{0} e_{2}$. There exists a $g_{0} \in L^{1}(G)$ with car $\widehat{g}_{0}-\operatorname{car} \widehat{g}_{0} \subset U$ and $\sigma\left(g_{0}\right) x_{0}$ $\neq 0$. Put $y_{0} \equiv \sigma\left(g_{0}\right) x_{0}$. Then $e_{1} y_{0} e_{2}=y_{0}$ and $\operatorname{sp}_{\sigma}\left(y_{0}\right)-\operatorname{sp}_{\sigma}\left(y_{0}\right) \subset U$. Let $f_{1}^{o}$ $\equiv \sup \left\{\mathrm{s}\left(\sigma_{t}\left(y_{0}{ }^{*}\right)\right): t \in G\right\}$ and $f_{2}{ }^{o} \equiv \sup \left\{\mathrm{s}\left(\sigma_{t}\left(y_{0}\right)\right): t \in G\right\}$. Then we have projections $f_{j}^{o}$ in $M^{\sigma}$ such that $0<f_{j}^{o} \leq e_{j}$ for $j=1,2$.

Let $\mathscr{I}$ be the family of sets of $\left(x_{\alpha}, g_{\alpha}\right) \in M \times L^{1}(G)$ such that

(a) $x_{\alpha}=e_{1} x_{\alpha} e_{2} \neq 0$;

(b) $\operatorname{car} \widehat{g}_{\alpha}-\operatorname{car} \widehat{g}_{\alpha} \subset U$; and

(c) projections $\bar{f}_{1}^{\alpha}$ are mutually orthogonal, where $f_{1}^{\alpha} \equiv \sup \left\{\mathrm{s}\left(\sigma_{t}\right.\right.$ $\left.\left.\left(y_{\alpha}^{*}\right)\right): t \in G\right\}$ and $y_{\alpha} \equiv \sigma\left(g_{\alpha}\right) x_{\alpha}$.

Since $\mathscr{I}$ is ordered by set inclusion, we have a maximal set $F \in \mathscr{F}$ by Zorn's lemma, say $F=\left\{\left(x_{\alpha}, g_{\alpha}\right) \in M \times L^{1}(G)\right.$ : (a), (b), (c) $\}$. By maximality, $\sum \bar{f}_{1}{ }^{\alpha}=1$. Let $f_{2}{ }^{\alpha} \equiv \sup \left\{\mathrm{s}\left(\sigma_{t}\left(y_{\alpha}\right)\right): t \in \mathrm{G}\right\}$ and $f_{j} \equiv \sum f_{j}^{\alpha}$ for $j=1,2$. Since $\mathrm{s}\left(\sigma_{t}\left(y_{\alpha}^{*}\right)\right) \sim \mathrm{s}\left(\sigma_{t}\left(y_{\alpha}\right)\right)$ in $M$ for each $t \in G$, we have $\bar{f}_{1}^{\alpha}$ $=\bar{f}_{2}^{\alpha}$ and $\bar{f}_{1}=\bar{f}_{2}=1$.

Suppose that $\gamma \in \operatorname{sp} \sigma^{f_{1}}$. For any compact neighbourhood $V$ of $\gamma$ there exists a non zero $x$ in $M^{\sigma}(V)$ with $x=f_{1}^{\alpha} x f_{1}^{\alpha}$ for some $\alpha$. Since $x=f_{1}^{\alpha} x f_{1}^{\alpha}$, it follows that $\sigma_{t_{2}}\left(y_{\alpha}{ }^{*}\right) x \sigma_{t_{1}}\left(y_{\alpha}\right) \neq 0$ for some $t_{1}$ and $t_{2}$ in $G$. Put $y \equiv \sigma_{t_{2}}\left(y_{\alpha}{ }^{*}\right) x \sigma_{t_{1}}\left(y_{\alpha}\right)$. Since $\operatorname{sp}_{\sigma}(y) \subset V-U$ and $y=f_{2} y f_{2}$, we have $M^{\sigma}(V-U) \cap M_{f_{2}} \neq\{0\}$. Since $V \cap\left(U+\operatorname{sp} \sigma^{f_{2}}\right) \neq \emptyset$ and $U+\operatorname{sp} \sigma^{f_{2}}$ is closed, $\gamma \in U+\operatorname{sp} \sigma^{f_{2}}$.

The remaining inclusion is proved similarly as above. Q.E.D. 
Lemma 3.2. $\Gamma_{1}\left(\sigma^{e}\right)=\Gamma_{1}\left(\sigma^{\bar{e}}\right)$ for $e \in M^{\sigma}$.

Lemma 3.3. Let $\mathscr{I}(\sigma)$ be the set of all $\operatorname{sp} \sigma^{e}+V$ for $e$ in $M^{\sigma}$ (or $Z\left(M^{\sigma}\right)$ ) with $\bar{e}=1$ and compact neighbourhoods $V$ of 0 in $\widehat{G}$. Then $\mathscr{I}(\sigma)$ is a filter base and $\Gamma_{1}(\sigma)=\cap\{F: F \in \mathscr{F}(\sigma)\}$.

These two lemmas are proved by combining Lemma 3.1 and similar arguments as the proofs of [6, Lemmas 2.3.3 and 2.3.4].

We are now ready to give a sufficient condition for a problem of Borchers which is proposed in the final remark in [4].

Proof of the implication (C) $\Rightarrow(\mathrm{A})$ in (ii). Since $\Gamma_{0}(\sigma)=\Xi$, it follows that $\Xi \subset \mathrm{sp} \sigma^{e}$ for all non zero $e$ in $M^{\sigma}$ (or $Z\left(M^{\sigma}\right)$ ).

Suppose that $f$ is a non zero projection in $M^{\sigma}$ (or $Z\left(M^{\sigma}\right)$ ). For any $\varepsilon$ in $(0,1)$ and $t_{j} \in \Xi^{\perp}$ for $j=1,2, \cdots, n, V$ denotes the set of $\gamma \in \widehat{G}$ such that $1-\varepsilon<\operatorname{Re}\left\langle t_{j}, \hat{\imath}\right\rangle$ for all $j=1,2, \cdots, n$. Let $\phi$ be the quotient mapping of $\widehat{G}$ onto $\widehat{G} / \Xi$.

Since $\Gamma_{0}(\sigma) \subset \Gamma_{0}\left(\sigma^{f}\right) \subset \Gamma_{1}\left(\sigma^{f}\right)=\Gamma_{1}\left(\sigma^{f}\right) \subset \Gamma_{1}(\sigma)$, we have $\Gamma_{0}\left(\sigma^{f}\right)=\Gamma_{1}\left(\sigma^{f}\right)$ $=\Xi$. By restricting our argument to $M_{f}$, we may assume that $f=1$ for the moment. Since $\mathscr{I}(\sigma)$ in Lemma 3.3 is a filter base and $\widehat{G} / \Xi$ is compact, $\{\phi(F): F \in \mathscr{F}(\sigma)\}$ is also a filter base of compact sets. Since $t_{j} \in \Xi^{\perp}$ for $j=1,2, \cdots, n$, we have $V+\Xi=V$ and hence $\phi^{-1}(\phi(V))=V$. Since $\operatorname{sp} \sigma$ $+\Gamma_{0}(\sigma)=\operatorname{sp} \sigma$, we have $\phi^{-1}(\phi(F))=F$ for all $F \in \mathscr{F}(\sigma)$. Hence Lemma 3.3 implies that the intersection of all $\phi(F), F \in \mathscr{F}(\sigma)$ is zero. Since $\widehat{G} / \Xi$ is compact, $\phi(F)$ converges to 0 and hence there exists an $F \in \mathscr{I}(\sigma)$ such that $\phi(F) \subset \phi(V)$ or $F \subset V$.

Consequently, sp $\sigma^{e} \subset V$ for some non zero $e$ in $M^{\sigma}\left(\right.$ or $\left.Z\left(M^{\sigma}\right)\right)$ with $e \leq f$

Q.E.D.

The case $\Xi=\{0\}$ is a special case where $\Xi^{\perp}$ is not discrete.

Making a slight modification of [13, Theorem 5.2], we have the following lemma.

Lemma 3.4. If $G$ satisfies the second axiom of countability, a Borel multiplier $\alpha \in Z^{2}(G, \boldsymbol{T})$ with $\alpha(s, t)=\alpha(t, s)$ for $s, t \in G$ is triv- 
ial, namely, $\alpha \in B^{2}(G, T)$.

Proof. Let $G^{\alpha} \equiv G \times \boldsymbol{T}$ be the extension of $G$ by $\alpha$, that is, the product is defined by

$$
\left(t_{1}, \lambda_{1}\right)\left(t_{2}, \lambda_{2}\right) \equiv\left(t_{1}+t_{2}, \alpha\left(t_{1}, t_{2}\right) \lambda_{1} \lambda_{2}\right)
$$

for $\left(t_{j}, \lambda_{j}\right) \in G^{\alpha} . \quad G^{\alpha}$ is given the product Borel structure of $G \times \mathbf{T}$. Since $G$ satisfies the second axiom of countability and $\alpha(s, t)=\alpha(t, s)$ for $s$, $t \in G, G^{\alpha}$ is a locally compact abelian group with respect to the Weil topology. Let $j$ be an injection of $\boldsymbol{T}$ to $G^{\alpha}$ such that $j(\lambda)=(0, \lambda)$ for $\lambda \in \boldsymbol{T}$. Since $j(\mathbb{T})$ is a topological subgroup of $G^{\alpha}$ which is standard and $j$ is a Borel measure isomorphism, $j$ is a homeomorphism by a Mackey's theorem [13, Theorem 2.2]. Let $\widehat{G}^{\alpha}$ and $\widehat{j(\boldsymbol{T})}$ denote the duals of $G^{\alpha}$ and $j(\boldsymbol{T})$, respectively. Let $l$ be a mapping of $j(\boldsymbol{T})$ to $\boldsymbol{T}$ such that $l:(0, \lambda) \in j(\boldsymbol{T}) \rightarrow \lambda \in \boldsymbol{T}$. Then $l \in \widehat{j(\boldsymbol{T})}$. Since $\widehat{G}^{\alpha} / j(\boldsymbol{T})^{\perp}$ is isomorphic to $\widehat{j(\boldsymbol{T})}$, we have the corresponding $\chi^{*} \in \widehat{G}^{\alpha} / j(\boldsymbol{T})^{\perp}$ to $l \in \widehat{j(\boldsymbol{T})}$. If $\chi \in \chi^{*}$, then $\chi \in \widehat{G}^{\alpha}$ and $\chi=l$ on $j(\boldsymbol{T})$. Put $\beta(t) \equiv \chi((t, 1))$. From $(3 \cdot 1)$ we have $\beta\left(t_{1}\right) \beta\left(t_{2}\right)=\beta\left(t_{1}+t_{2}\right) \alpha\left(t_{1}, t_{2}\right)$.

Q.E.D.

Remark 3.1. This lemma is partly generalized as the following. Every symmetric (i.e., $\alpha(s, t)=\alpha(t, s)$ ) multiplier is trivial for an abelian discrete group. For this we have only to assume the product topology on $G^{\alpha}=G \times \boldsymbol{T}$ in the above proof.

Proof of (iii) in Theorem 1.1. We have only to prove that the condition (B) implies the condition (A). Since $0 \in \operatorname{sp}_{\sigma_{e}}\left(1_{e}\right)$ for any non zero $e$ in $M^{\sigma}$ (or $Z\left(M^{\sigma}\right)$ ), we have $\Xi=\{0\} \subset \operatorname{sp} \sigma^{e}$.

Suppose that $V$ is a neighbourhood of $\Xi$. Since $0 \in \Xi$, we may choose an open neighbourhood $U$ of 0 with $(U-U)^{-} \subset V$. Since $G(\sigma)$ $=G$ and $M_{*}$ is separable, it follows from Lemma 3.4 and $[8,11,12]$ that there exists a strongly continuous unitary representation $u$ of $G$ in $M^{\sigma}$ such that $\sigma_{t}(x)=u_{t} x u_{t}^{*}$ for $x \in M$ and $t \in G$. By virtue of Stone's theorem, we have a spectral resolution

$$
u_{t}=\int_{\Gamma} \overline{\langle t, \gamma\rangle} e(d \gamma)
$$


where $e(d \gamma)$ is a spectral projection measure on $\widehat{G}$. Utilizing a $\gamma_{0} \in \widehat{G}$ with $e\left(U+\gamma_{0}\right) f \neq 0$, we define a projection $e$ by $e\left(U+\gamma_{0}\right) f$. Then $e \in M^{\sigma}$ (or $Z\left(M^{\sigma}\right)$ ) and $0<e \leq f$. For all $g \in L^{1}(G)$ with car $\widehat{g} \subset \widehat{G} \backslash(U-U)$ we have

$$
\begin{aligned}
e(\sigma(g) x) e & =\int_{G} g(t) e u_{t} x u_{t}^{*} e d t \\
& =\int_{U} \int_{U} \hat{g}\left(\gamma-\gamma^{\prime}\right) e(d \gamma) x e\left(d \gamma^{\prime}\right)=0
\end{aligned}
$$

for all $x \in M$. Therefore $\operatorname{sp} \sigma^{e} \subset(U-U)^{-} \subset V$.

Q.E.D.

Proof of Corollary 1.1. It is immediate from (iii) of Theorem 1.1.

The following proposition generalizes [6, Theorem 2.4.1].

Proposition 3.1. (i) If $Z\left(M^{\sigma}\right) \subset Z(M)$, then $\operatorname{sp} \sigma=\Gamma_{1}(\sigma)$. In particular, if $M^{\sigma}$ is a factor, $\Gamma_{0}(\sigma)=\Gamma_{1}(\sigma)$.

(ii) If $\Gamma_{0}(\sigma)=\Gamma_{1}(\sigma)=\Xi$ and $\Xi$ is discrete, then $\mathrm{sp} \sigma=\Xi$ is necessary and sufficient for $Z\left(M^{\sigma}\right) \subset Z(M)$.

Proof. (i) Let $e \in M^{\sigma}$ and $f$ the carrier in $Z\left(M^{\sigma}\right)$. If $\gamma \in \operatorname{sp} \sigma^{f}$, there exists an $x \in M$ such that $\operatorname{sp}_{\sigma}(x) \cap(V+\gamma) \neq \emptyset$ for any neighbourhood $V$ of 0 . Since $f$ is the carrier in $Z\left(M^{\sigma}\right)$ of $e$, there exist $y$ and $z$ in $M^{\sigma}$ with $e z x y e \neq 0$. Since $\operatorname{sp}_{\sigma}(e z x y e)=\operatorname{sp}_{\sigma}(x), \quad \gamma \in \operatorname{sp} \sigma^{e}$ and hence $\operatorname{sp} \sigma^{f}$ $\subset \operatorname{sp} \sigma^{e}, \quad$ which implies $\operatorname{sp} \sigma^{e}=\operatorname{sp} \sigma^{f}$. Consequently, $\Gamma_{1}(\sigma)=\cap\left\{\operatorname{sp} \sigma^{f}:\right.$ $f \in Z\left(M^{\sigma}\right)$ and $\left.\bar{f}=1\right\}$. Since $Z\left(M^{\sigma}\right) \subset Z(M)$, we have sp $\sigma=\Gamma_{1}(\sigma)$.

(ii) By (i) we have only to show the sufficiency. First we shall show that if $e_{1}$ and $e_{2}$ in $M^{\sigma}$ have mutually orthogonal central carriers, then $\bar{e}_{1} \bar{e}_{2}=0$. Suppose that $e_{1} \in M^{\sigma}, e_{2} \in M^{\sigma}$ and $\bar{e}_{1} \bar{e}_{2} \neq 0$. Then there exists a non zero $x \in M$ such that $e_{1} x e_{2}=x$ and $\operatorname{sp}_{\sigma}(x)=\{\gamma\}$ for some $\gamma \in \Xi$. Put $e_{3} \equiv \mathrm{s}(x)$. Since $\Gamma_{1}\left(\sigma^{e_{3}}\right)=\Xi$ by Lemma 3.2 and since $\Gamma_{1}\left(\sigma^{e_{3}}\right)$ $=\operatorname{sp} \sigma^{e_{3}}$ from the assumption that $\Xi=\operatorname{sp} \sigma$, it follows that there exists a non zero $y \in M_{e_{3}}$ with $\operatorname{sp}_{\sigma}(y)=\{-\gamma\}$. Then $e_{1} x y e_{2}=x y \neq 0$ and $x y \in M^{\sigma}$. Thus the product of central carriers of $e_{1}$ and $e_{2}$ is non zero.

Now, suppose $e \in Z\left(M^{\sigma}\right)$. Since $e(1-e)=0$, we have $\bar{e} \overline{(1-e)}=0$ from the above. Since $1=e+(1-e) \leq \bar{e}+\overline{(1-e)} \leq 1$, we have $e=\bar{e}$, 
namely, $e \in Z(M)$.

Q.E.D.

Remark 3.2. Assume that $\widehat{G} / \Xi$ is compact. If $M^{\sigma}$ is a factor, then the conditions (A), (B) and (C) are equivalent. For this we have only to show that (B) implies (C). Since $G(\sigma)$ is discrete by assumption, there exists by Lemma 4.4 a $\sigma^{\prime} \in \operatorname{Rep}(G, M)$ such that $\sigma^{\prime} \sim \sigma, Z\left(M^{\sigma^{\prime}}\right)$ $=Z\left(M^{\sigma}\right)$ and $G(\sigma)=\left(\operatorname{sp} \sigma^{\prime}\right)^{\perp}$. If $M^{\sigma}$ is a factor, then $M^{\sigma^{\prime}}$ is a factor and hence $\Gamma_{0}\left(\sigma^{\prime}\right)=\Gamma_{1}\left(\sigma^{\prime}\right)=\operatorname{sp} \sigma^{\prime}$ by Proposition 3.1. Therefore $\operatorname{sp} \sigma^{\prime}$ is a group and hence $\Xi=G(\sigma)^{\perp}=\operatorname{sp} \sigma^{\prime}=\Gamma_{1}\left(\sigma^{\prime}\right)$ by $(\mathrm{B})$. Since $\Gamma_{0}(\sigma)=\Gamma_{1}(\sigma)$ by Proposition 3.1 and $\Gamma_{1}(\sigma)=\Gamma_{1}\left(\sigma^{\prime}\right)$ by Lemma 4.3, we have $\Gamma_{0}(\sigma)$ $=\Gamma_{1}(\sigma)=\Xi$, which is $(\mathrm{C})$.

\section{§4. Proof of $(B) \Rightarrow(C)$ and S-Set}

In the following $I_{\varepsilon}(\lambda)$ denotes the $\varepsilon$-neighbourhood of $\lambda \in \boldsymbol{R}$, namely, the open interval $(\lambda-\varepsilon, \lambda+\varepsilon)$.

Proposition 4.1. For a $\sigma \in \operatorname{Rep}(\boldsymbol{R}, M)$ there exist projections $q_{0}$, $q_{\infty}$ in $Z\left(M^{\sigma}\right) \cap Z(M)$ and an increasing left continuous spectral resolution $\left\{p(\lambda) \in Z\left(M^{\sigma}\right) \cap Z(M): \lambda>0\right\}$ of $q_{\infty}-q_{0}$ such that

(i) $\Gamma_{0}\left(\sigma^{q_{0}}\right)=\Gamma_{1}\left(\sigma^{q_{0}}\right)=\{0\}$;

(ii) $\Gamma_{0}\left(\sigma^{1-q_{\infty}}\right)=\Gamma_{1}\left(\sigma^{1-q_{\infty}}\right)=\boldsymbol{R}$;

(iii) for any non zero $\lambda \in \operatorname{Sp} h\left(h \equiv \int \lambda p(d \lambda)\right), \varepsilon_{0} \in(0, \lambda)$ and $\varepsilon \in(0$, $\left.\varepsilon_{0}\right)$ there exists a non zero projection $e \in Z\left(M^{\sigma}\right)$ majorized by $p\left(\lambda+\varepsilon_{0}\right)$ $-p\left(\lambda-\varepsilon_{0}\right)$ satisfying that $\operatorname{sp} \sigma^{f} \subset I_{\varepsilon}(\lambda) Z \cup I_{\varepsilon}(0)$ and $\operatorname{sp} \sigma^{f} \cap n I_{\varepsilon}(\lambda) \neq \emptyset$ for all $n \in \boldsymbol{N}$ and $f \in Z\left(M^{\sigma}\right)$ with $0<f \leq e$;

(iv) if $\lambda \in \mathrm{Sp} h$ and $\lambda>0$, then $\lambda Z \subset \Gamma_{1}(\sigma)$; and

(v) if $\lambda \in \mathrm{Sp} h$ is isolated and $q_{\lambda} \equiv p(\lambda+0)-p(\lambda)$, then $\Gamma_{0}\left(\sigma^{q_{\lambda}}\right)$ $=\Gamma_{1}\left(\sigma^{q_{\lambda}}\right)=\lambda Z$.

Proof. (i) Let $\mathscr{F}_{0}$ be the set of all projections $p \in Z\left(M^{\sigma}\right) \cap Z(M)$ such that for any $e \in Z\left(M^{\sigma}\right) \cap Z(M)$ with $0<e \leq p$ and for any $\lambda>0$ and $\delta \in(0, \lambda / 2)$ there exists a projection $f \in Z\left(M^{\sigma}\right)$ with $0<f \leq e$ and $\operatorname{sp} \sigma^{f} \cap$ $(\delta, \lambda-\delta)=\emptyset$. Put $q_{0} \equiv \sup \left\{p: p \in \mathscr{F}_{0}\right\}$. Using Zorn's lemma, we have a projection $e_{0} \in Z\left(M^{\sigma}\right)$ such that $\bar{e}_{0}=q_{0}$ and $\operatorname{sp} \sigma^{e_{0}} \cap(\delta, \lambda-\delta)=\emptyset$. 
Therefore

$$
\Gamma_{1}\left(\sigma^{q_{0}}\right) \subset \bigcap_{\lambda>0,0<\delta<\lambda / 2} \bigcap_{R} \boldsymbol{R} \backslash\{(-\lambda+\delta,-\delta) \cup(\delta, \lambda-\delta)\}=\{0\},
$$

and hence $\Gamma_{0}\left(\sigma^{q_{0}}\right)=\Gamma_{1}\left(\sigma^{q_{0}}\right)=\{0\}$.

(ii) Let $\mathscr{F}_{\infty}$ be the set of all projections $e \in Z\left(M^{\sigma}\right)$ such that $\operatorname{sp} \sigma^{e}$ $\neq \boldsymbol{R}$. Put $q_{\infty} \equiv \sup \left\{\bar{e}: e \in \mathscr{F}_{\infty}\right\}$. Then $q_{0} \leq q_{\infty}$ and $\Gamma_{0}\left(\sigma^{1-q_{\infty}}\right)=\Gamma_{1}\left(\sigma^{1-q_{\infty}}\right)$ $=\boldsymbol{R}$. Moreover, $q_{\infty} \in Z\left(M^{\sigma}\right) \cap Z(M)$.

For the proof of the remaining part we must prepare the following two lemmas. Before going into the proof we recall that if $\operatorname{sp} \sigma \cap(\delta, \lambda-\delta)$ $\neq \emptyset$ with $0<2 \delta<\lambda$ then for any $\varepsilon>0$ there exist a $\lambda_{0} \in(\delta, \lambda-\delta)$ and a non zero $x \in M$ such that

$$
\operatorname{sp}_{\sigma}(x) \subset I_{\varepsilon}\left(\lambda_{0}\right) \cap(\delta, \lambda-\delta) .
$$

Lemma 4.1. Assume that $q_{0}=1-q_{\infty}=0$. For any $\lambda>0$ and $\delta$ $\in(0, \lambda / 2)$ let $\mathscr{F}_{\lambda, \delta}$ be the set of all projections $e$ in $Z\left(M^{\sigma}\right) \cap Z(M)$ such that if $f$ is a projection in $Z\left(M^{\sigma}\right)$ with $0<f \leq e$ then $\operatorname{sp} \sigma^{f} \cap(\delta, \lambda-\delta)$ $\neq \emptyset$. If

$$
p(\lambda) \equiv \sup _{0<2 \delta<\lambda} \sup \left\{e: e \in \mathscr{F}_{\lambda, \delta}\right\}
$$

then $\{p(\lambda): \lambda>0\}$ is an increasing and left continuous spectral resolution of the identity.

Proof. In the following we denote $\sup \left\{e: e \in \mathscr{F}_{\lambda, \delta}\right\}$ by $p(\lambda, \delta)$.

Since $p(\lambda, \delta) \leq p(\mu, \delta)$ for $0<\lambda \leq \mu$, it follows that $p(\lambda) \leq p(\mu)$ for $0<\lambda \leq \mu$. Therefore $p(\lambda)$ is increasing in $\lambda>0$.

Since $\mathscr{F}_{\lambda, \varepsilon+\hat{o}} \subset \mathscr{F}_{\lambda-\varepsilon, \delta}$, it follows that

$$
\begin{aligned}
\lim _{\varepsilon \downarrow 0} p(\lambda-\varepsilon) & =\sup _{\varepsilon>0} p(\lambda-\varepsilon)=\sup _{\varepsilon>0} \sup _{\delta>0} p(\lambda-\varepsilon, \delta) \\
& \geq \sup _{\varepsilon>0} \sup _{\delta>0} p(\lambda, \varepsilon+\delta)=p(\lambda),
\end{aligned}
$$

and hence that $p(\lambda)$ is left continuous.

Putting $p_{\infty} \equiv 1-\lim _{\lambda \rightarrow \infty} p(\lambda)$, we have $p_{\infty} \in Z\left(M^{\sigma}\right) \cap Z(M)$ and

$$
p_{\infty}=\inf _{\lambda>0} \inf _{\delta>0}(1-p(\lambda, \delta)) \text {. }
$$


Suppose that $p_{\infty} \neq 0$. If $e$ is a projection in $Z\left(M^{\sigma}\right) \cap Z(M)$ with $0<e \leq p_{\infty}$, then for any $\lambda>0$ and $\delta \in(0, \lambda / 2)$ there exists a projection $f \in Z\left(M^{\sigma}\right)$ with $0<f \leq e$ and $\operatorname{sp} \sigma^{\varsigma} \cap(\delta, \lambda-\delta)=\emptyset$. Thus $p_{\infty} \leq q_{0}$. Since $q_{0}=0$ by assumption, it follows that $\lim _{\lambda \rightarrow \infty} p(\lambda)=1$.

Putting $p_{0} \equiv \lim _{\lambda \rightarrow 0} p(\lambda)$, we have $p_{0} \in Z\left(M^{\sigma}\right) \cap Z(M)$ and

$$
p_{0}=\inf _{\lambda>0} \sup \{p(\lambda, \delta): 0<2 \delta<\lambda\} .
$$

Suppose that $p_{0} \neq 0$. If $e$ is a projection in $Z\left(M^{\sigma}\right)$ with $0<e \leq p_{0}$, then for any $\lambda>0$ there exist a $\delta \in(0, \lambda / 2)$ and a projection $e_{0} \in Z\left(M^{\sigma}\right), 0<e_{0}$ $\leq e p(\lambda, \delta)$ such that $\operatorname{sp} \sigma^{f} \cap(\delta, \lambda-\delta) \neq \emptyset$ whenever $f \in Z\left(M^{\sigma}\right)$ and $0<f$ $\leq e_{0}$.

For any $\mu>0$ and its $\varepsilon$-neighbourhood $I_{\varepsilon}(\mu) \subset \boldsymbol{R}_{+}$we shall show $I_{\varepsilon}(\mu) \cap \operatorname{sp} \sigma^{e}$ is non empty. For a given $\varepsilon$ we have a positive $\lambda<\varepsilon$, for which we get a $\delta$ and a projection $e_{0}$ as above. Choose an $n \in \boldsymbol{N}$ so that $\mu \leq n \delta$ and put $\eta \equiv \delta / n$. Since $\operatorname{sp} \sigma^{e_{0}} \cap(\delta, \lambda-\delta) \neq \emptyset$, we have a non zero $x_{1} \in M_{e_{0}}$ and a $\lambda_{1} \in(\delta, \lambda-\delta)$ satisfying

$$
\operatorname{sp}_{\sigma}\left(x_{1}\right) \subset I_{\eta}\left(\lambda_{1}\right) \cap(\delta, \lambda-\delta) .
$$

Let $f_{1}$ be the carrier in $Z\left(M^{\sigma}\right)$ of

$$
\sup \left\{\sigma_{t}\left(s\left(x_{1}\right)\right): t \in \boldsymbol{R}\right\},
$$

where $\mathrm{s}\left(x_{1}\right)$ denotes the carrier of $x_{1}$. Since $f_{1} \in Z\left(M^{\sigma}\right)$ and $0<f_{1} \leq e_{0}$, we have $\operatorname{sp} \sigma^{f_{1}} \cap(\delta, \lambda-\delta) \neq \emptyset$. Therefore we have a non zero $x_{2} \in M_{f_{1}}$ and a $\lambda_{2} \in(\delta, \lambda-\delta)$ satisfying $\operatorname{sp}_{\sigma}\left(x_{2}\right) \subset I_{\eta}\left(\lambda_{2}\right) \cap(\delta, \lambda-\delta)$. Since $x_{2} \in M_{f_{1}}$, we have a $v_{1} \in M^{\sigma}$ and a $t_{1} \in \mathbb{R}$ with

$$
y_{2} \equiv \sigma_{t_{1}}\left(x_{1}\right) v_{1} x_{2} \neq 0 \text {. }
$$

Let $f_{2}$ be the carrier in $Z\left(M^{\sigma}\right)$ of

$$
\sup \left\{\sigma_{t}\left(\mathrm{~s}\left(y_{2}\right)\right): t \in \boldsymbol{R}\right\} .
$$

Since $f_{2} \in Z\left(M^{\sigma}\right)$ and $0<f_{2} \leq e_{0}$, we have $\operatorname{sp} \sigma^{f_{2}} \cap(\delta, \lambda-\delta) \neq \emptyset$. We repeat the similar argument as above and obtain sets $\left\{x_{1}, \cdots, x_{n}\right\} \subset M$ and $\left\{\lambda_{1}, \cdots, \lambda_{n}\right\} \subset(\delta, \lambda-\delta)$ satisfying

$$
\operatorname{sp}_{\sigma}\left(x_{j}\right) \subset I_{\eta}\left(\lambda_{j}\right) \cap(\delta, \lambda-\delta)
$$

for $j=1, \cdots, n$. Since $f_{j}$ is the carrier in $Z\left(M^{\sigma}\right)$ of 


$$
\sup \left\{\sigma_{t}\left(\mathrm{~s}\left(y_{j}\right)\right): t \in \boldsymbol{R}\right\}
$$

and $x_{j+1} \in M_{f_{j}}$ for $j=2, \cdots, n$, we have sets $\left\{v_{1}, \cdots, v_{n-1}\right\} \subset M^{\sigma}$ and $\left\{t_{1}, \cdots\right.$, $\left.t_{n-1}\right\} \subset \boldsymbol{R}$ satisfying

$$
y_{k} \equiv \sigma_{t_{k-1}}\left(\cdots \sigma_{t_{2}}\left(\sigma_{t_{1}}\left(x_{1}\right) v_{1} x_{2}\right) v_{2} x_{3} \cdots\right) v_{k-1} x_{k} \neq 0
$$

for all $k=2, \cdots, n$. Since $\operatorname{sp}_{\sigma}\left(y_{k}\right) \subset\left\{\sum_{j=1}^{k} \operatorname{sp}_{\sigma}\left(x_{j}\right)\right\}^{-}$, we have

$$
\operatorname{sp}_{\sigma}\left(y_{k}\right) \subset \sum_{j=1}^{k} I_{\eta}\left(\lambda_{j}\right) \subset I_{n \eta}\left(\sum_{j=1}^{k} \lambda_{j}\right) .
$$

Since $\mu \leq n \delta<\sum_{j=1}^{n} \lambda_{j}, \delta<\lambda_{j}<\lambda-\delta$ and $\lambda<\varepsilon$, there exists an $m \in N, m<n$ with $\mu-\varepsilon<\sum_{j=1}^{m} \lambda_{j}<\mu$. Since $\delta=n \eta$, we have $I_{\delta}\left(\sum_{j=1}^{m+1} \lambda_{j}\right) \subset I_{\varepsilon}(\mu)$, and hence

$$
y_{m+1} \in M_{e_{0}} \subset M_{e} \quad \text { and } \quad \operatorname{sp}_{\sigma}\left(y_{m+1}\right) \subset I_{\varepsilon}(\mu) .
$$

Since $\varepsilon$ can be arbitrarily small, it follows that $\mu \in \operatorname{sp} \sigma^{e}$. Since $\mu(>0)$ is arbitrary, $\operatorname{sp} \sigma^{e}=\boldsymbol{R}$. The arbitrariness of $e \in Z\left(M^{\sigma}\right)$ with $0<e \leq p_{0}$ implies that $\Gamma_{0}\left(\sigma^{p_{0}}\right)=\boldsymbol{R}$. Since $q_{\infty}=1$ by assumption, we have a contradiction. Thus $p_{0}=0$, namely, $\lim _{\lambda \rightarrow 0} p(\lambda)=0$ Q.E.D.

The idea of the following lemma is essentially due to Borchers, [4].

Lemma 4.2. For any $\varepsilon \in(0, \lambda / 2)$ let $p$ be a non zero projection in $Z\left(M^{\sigma}\right)$ satisfying $\operatorname{sp} \sigma^{e} \cap I_{\varepsilon}(\lambda) \neq \emptyset$ for all $e \in Z\left(M^{\sigma}\right)$ with $0<e \leq p$. For a non zero projection $q$ in $Z\left(M^{\sigma}\right)$ if $l(q)$ is defined by

$$
\sup \left\{k(e): e \in Z\left(M^{\sigma}\right), 0<e \leq q\right\},
$$

where $k(e)$ denotes the supremum length of subintervales of $(0, \lambda+\varepsilon)$ $\backslash \mathrm{sp} \sigma^{e}$, then

(i) $\operatorname{sp} \sigma^{e} \cap n I_{\varepsilon}(\lambda) \neq \emptyset$ for all $e \in Z\left(M^{\sigma}\right)$ with $0<e \leq p$ and $n \in Z$; and

(ii) for any $\delta>0$ there exists a projection $e$ in $Z\left(M^{\sigma}\right)$ such that $0<e \leq p$ and $\operatorname{sp} \sigma^{e} \subset I_{\delta}(l(p)) \boldsymbol{Z} \cup I_{\delta}(0)$.

Proof. (i) We shall use an induction argument. Suppose that $\operatorname{sp} \sigma^{e}$ $\cap n I_{\varepsilon}(\lambda) \neq \emptyset$ for some $n>0$. For a non zero $x$ in $M^{\text {oe }}\left(n I_{\varepsilon}(\lambda)\right)$ let $f$ $\equiv \sup \left\{\sigma_{t}(\mathrm{~s}(x)): t \in G\right\}$. Then $0<f \leq e \leq p$. Since $\operatorname{sp} \sigma^{f} \cap I_{\varepsilon}(\lambda) \neq \emptyset$ by as- 
sumption, we have a non zero $y$ in $M^{\sigma f}\left(I_{\varepsilon}(\lambda)\right)$. Since $0<f \leq e$ and $\sigma_{t}^{e}(x) y \neq 0$ for some $t \in G$, we have $\operatorname{sp} \sigma^{e} \cap(n+1) I_{\varepsilon}(\lambda) \neq \emptyset$.

(ii) We have nothing to prove if $\delta>2^{-1} l(p)$. For any positive $\delta \leq 2^{-1} l(p)$ there exists a projection $e \in Z\left(M^{\sigma}\right)$ such that $0<e \leq p$ and $k(e)>l(p)-\delta$. Put $l \equiv l(p)$. We have then a subinterval $\left(2^{-1} \delta, l-2^{-1}\right.$ $\cdot \delta)+\lambda_{0}$ of $(0, \lambda+\varepsilon) \backslash \operatorname{sp} \sigma^{e}$ for some $\lambda_{0} \in \boldsymbol{R}$. We shall show by induction that $(n-1) l+n \delta, n(l-\delta)) \cap \operatorname{sp} \sigma^{e}=\emptyset$ for all $n \in \mathbf{N}$ with $n<(2 \delta)^{-1} l$. For $n=1$ we assume the contrary. Let $\phi(E, e)$ for $e \in M^{\sigma}$ denote the projection onto the subspace spanned by $M^{\sigma}(E) e \mathcal{H}$. For any $\lambda \in(\delta$, $l-\delta) \cap \operatorname{sp} \sigma^{e}$ we have

$$
e^{\prime} \equiv e \phi\left(\left[-\delta^{\prime}, \delta^{\prime}\right]+\lambda, e\right)
$$

for $\delta^{\prime} \in(0, \min \{\lambda-\delta, l-\delta-\lambda\})$. Since $\lambda \in \operatorname{sp} \sigma^{e}$, we have $e^{\prime} \neq 0$ and $\operatorname{sp} \sigma^{e^{\prime}}$ $\subset \operatorname{sp} \sigma^{e} \cap\left(\operatorname{sp} \sigma^{e}-I_{\delta^{\prime}}(\lambda)^{-}\right)$, for $\operatorname{sp}^{e} \sigma^{\phi(E, e)} \subset \operatorname{sp} \sigma^{e}-E$ with $E \equiv I_{\delta^{\prime}}(\lambda)^{-}$. Therefore $s_{\lambda} \sigma^{e^{\prime}}$ is disjoint from

$$
\begin{gathered}
\left(\lambda_{0}+2^{-1} \delta, \lambda_{0}+l-2^{-1} \delta\right) \cup\left(\lambda_{0}+2^{-1} \delta-\lambda+\delta^{\prime}, \lambda_{0}+l-2^{-1} \delta-\lambda-\delta^{\prime}\right) \\
=\left(\lambda_{0}-\lambda+2^{-1} \delta+\delta^{\prime}, \lambda_{0}+l-2^{-1} \delta\right)
\end{gathered}
$$

Since $\lambda_{0}+l-2^{-1} \delta>0$ and $0 \in \operatorname{sp} \sigma^{e^{\prime}}, \lambda_{0}-\lambda+2^{-1} \delta+\delta^{\prime}>0$. The length $l^{\prime}$ of the interval on the right hand side is $l-\delta+\lambda-\delta^{\prime}$. Since $l<l^{\prime} \leq l\left(e^{\prime}\right)$, we have a contradiction. Thus $\operatorname{sp} \sigma^{e}$ is disjoint from $(\delta, l-\delta)$. Suppose that the result is true for $n>1\left(n<(2 \delta)^{-1} l-1\right)$. If $(n l+(n+1) \delta,(n+1)$ $(l-\delta)) \cap \operatorname{sp} \sigma^{e} \neq \emptyset$, then

$$
f \equiv e \phi\left(\left[-\delta^{\prime \prime}, \delta^{\prime \prime}\right]+\mu, e\right)
$$

is non zero for any fixed

$$
\begin{aligned}
& \mu \in(n l+(n+1) \delta,(n+1)(l-\delta)) \cap \operatorname{sp} \sigma^{t} \\
& \delta^{\prime \prime} \in(0, \min \{\mu-n l-(n+1) \delta,(n+1)(l-\delta)-\mu\}) .
\end{aligned}
$$

Since $\mu \in \operatorname{sp} \sigma^{e}$, we have $f \neq 0$ and $\operatorname{sp} \sigma^{f} \subset \operatorname{sp} \sigma^{e} \cap\left(\operatorname{sp} \sigma^{e}-I_{\delta^{\prime \prime}}(\mu)^{-}\right)$. Therefore $\mathrm{sp} \sigma^{f}$ is disjoint frem

$$
\begin{gathered}
(-l+\delta,-\delta) \cup\left((n-1) l+n \delta-\mu+\delta^{\prime \prime}, n(l-\delta)-\mu-\delta^{\prime \prime}\right) \\
=\left((n-1) l+n \delta-\mu+\delta^{\prime \prime},-\delta\right),
\end{gathered}
$$

whose length is larger than $l$, for $\operatorname{sp} \sigma^{f}=-\operatorname{sp} \sigma^{f}$. This contradicts 
with the fact that $l(f) \leq l(p) \equiv l$. Thus the result is true for $n+1$.

Q.E.D.

Proof of Proposition 4.1. (Continued). By our previous proofs of (i) and (ii) we may assume that $q_{0}=1-q_{\infty}=0$ in the ramaining part of the proof.

(iii) Suppose that $\lambda$ is a non zero element of $\operatorname{Sp} h$. For any $\varepsilon \in(0$, $\left.\varepsilon_{0} / 3\right)$

$$
p \equiv p(\lambda+\varepsilon)-p(\lambda-\varepsilon)>0
$$

Since $p(\lambda+\varepsilon)=\sup _{\delta>0} p(\lambda+\varepsilon, \delta)$, there exists a projection $q$ in $Z\left(M^{\sigma}\right) \cap$ $Z(M)$ such that $q p(\lambda-\varepsilon)=0$ and $0<q \leq p(\lambda+\varepsilon, \delta)$ for some $\delta \in(0, \varepsilon / 2)$. Since $q p(\lambda-\varepsilon)=0$, there exists a projection $e^{\prime} \in Z\left(M^{\sigma}\right)$ with $0<e^{\prime} \leq q$ and $\operatorname{sp} \sigma^{e^{\prime}} \cap(\delta, \lambda-\varepsilon-\delta)=\emptyset$. On the other hand, since $0<q \leq p(\lambda+\varepsilon, \delta)$, if $f \in Z\left(M^{\sigma}\right)$ and $0<f \leq q$ then $\operatorname{sp} \sigma^{f} \cap(\delta, \lambda+\varepsilon-\delta) \neq \emptyset$. Therefore, if $f \in Z\left(M^{\sigma}\right)$ and $0<f \leq e^{\prime}$, then $\operatorname{sp} \sigma^{f} \cap I \neq \emptyset$ with $I \equiv\left(\lambda-2^{-1} 3 \varepsilon, \lambda+\varepsilon\right)$ and hence $\operatorname{sp} \sigma^{f} \cap n I \neq \emptyset$ by (i) in Lemma 4.2. Furthermore, we can define $l\left(e^{\prime}\right)$ by the same way as in Lemma 4.2. It follows from the above that $\lambda-2 \varepsilon<l\left(e^{\prime}\right)<\lambda+\varepsilon$. By virtue of (ii) in Lemma 4.2 we have a projection $e \in Z\left(M^{\sigma}\right)$ with $0<e \leq e^{\prime}$ and $\operatorname{sp} \sigma^{e} \subset I_{\varepsilon}\left(l\left(e^{\prime}\right)\right) Z \cup I_{\varepsilon}(0)$. Then for any $f \in Z\left(M^{\sigma}\right)$ with $0<f \leq e$ we have

$$
\operatorname{sp} \sigma^{f} \subset I_{\varepsilon}\left(l\left(e^{\prime}\right)\right) \boldsymbol{Z} \cup I_{\varepsilon}(0) \subset I_{3 \varepsilon}(\lambda) \boldsymbol{Z} \cup I_{3 \varepsilon}(0) .
$$

Considering $3 \varepsilon$ as $\varepsilon$, we have (iii).

(iv) Suppose that $\lambda \in \operatorname{Sp} h$ and $\lambda>0$. We shall use the same notation as in (iii). For any $\varepsilon \in\left(0, \varepsilon_{0}\right)$ let $\mathscr{F}$ be the set of all projections $e$ $\in Z\left(M^{\sigma}\right)$ satisfying the same condition as in (iii). Put $e_{\varepsilon} \equiv \sup \{e: e \in$ $\mathscr{F}\}$. For any $p \in Z\left(M^{\sigma}\right)$ with $\bar{p}=1$ we set $p_{\varepsilon} \equiv p \bar{e}_{\varepsilon}$. By means of Lemma 3.1 since $\bar{p}_{\varepsilon}=\bar{e}_{\varepsilon}$ there exist projections $e_{1}$ and $e_{2}$ in $Z\left(M^{\sigma}\right)$ such that $e_{1} \leq e_{\varepsilon}, e_{2} \leq p_{\varepsilon}, \bar{e}_{1}=\bar{e}_{2}=\bar{e}_{\varepsilon}$ and $\operatorname{sp} \sigma^{e_{1}} \subset \operatorname{sp} \sigma^{e_{2}}+I_{\varepsilon}(0)$. This inclusion relation and the condition in (iii) imply that $\left(\operatorname{sp} \sigma^{e_{2}}+I_{\varepsilon}(0)\right) \cap n I_{\varepsilon}(\lambda) \neq \emptyset$ for all $n \in \boldsymbol{N}$. Since $e_{2} \leq p$, we have

$$
\left(\operatorname{sp} \sigma^{p}+I_{\varepsilon}(0)\right) \cap n I_{\varepsilon}(\lambda) \neq \emptyset
$$

for all $n \in \boldsymbol{N}$. Since $\varepsilon$ is arbitrary, $\lambda \boldsymbol{Z} \subset \operatorname{sp} \sigma^{p}$ and hence $\lambda Z \subset \Gamma_{1}(\sigma)$. 
(v) Since $\lambda$ is isolated in $\operatorname{Sp} h, \operatorname{Sp} h \cap\left(\lambda-\varepsilon_{0}, \lambda+\varepsilon_{0}\right)=\{\lambda\}$ for some $\varepsilon_{0}>0$. For any $\varepsilon \in\left(0, \varepsilon_{0}\right)$ let $\mathscr{F}_{\alpha}$ be the family of sets of non zero projections $e^{\prime}$ in $Z\left(M^{\sigma}\right)$ with mutually orthogonal carriers in $Z(M)$ majorized by $q_{\lambda} \equiv p(\lambda+\varepsilon)-p(\lambda)$ satisfying that if $f \in Z\left(M^{\sigma}\right)$ and $0<f \leq e^{\prime}$ then $\operatorname{sp} \sigma^{f}$ $\subset I_{\varepsilon}(\lambda) \boldsymbol{Z} \cup I_{\varepsilon}(0)$ and $\operatorname{sp} \sigma^{f} \cap n I_{\varepsilon}(\lambda) \neq \emptyset$ for all $n \in \boldsymbol{N}$. Since $\mathscr{F}_{\lambda}$ is ordered by set inclusion and inductive, we have a maximal $\left\{e_{\iota}: \iota \in I\right\} \in \mathscr{F}_{\lambda}$ by Zorn's lemma. Put $p_{\lambda} \equiv \sup \left\{\bar{e}_{\iota}: \iota \in I\right\}$. If $q_{\lambda}-p_{\lambda} \neq 0$, there exists by (iii) a non zero projection $e^{\prime \prime} \in Z\left(M^{\sigma}\right)$ satisfying the same condition as $e_{\iota} \in \mathscr{F}_{\lambda}$ and $\bar{e}^{\prime \prime} \bar{e}_{\iota}=0$ for all $\iota \in I$, which contradicts with the maximality of $\mathscr{F}_{\lambda}$. Thus $q_{\lambda}=p_{\lambda}$. Putting $e \equiv \sup \left\{e_{\iota}: \iota \in I\right\}$, we have $\bar{e}=q_{\lambda}$ and $\operatorname{sp} \sigma^{e} \subset I_{\varepsilon}(\lambda) \mathbb{Z}$ $\cup I_{\varepsilon}(0)$. Since $\varepsilon$ is arbitrary, we have $\Gamma_{1}\left(\sigma^{q_{\lambda}}\right) \subset \lambda Z$.

Suppose that $\Gamma_{0}\left(\sigma^{q_{\lambda}}\right) \neq \lambda \boldsymbol{Z}$. Since $\Gamma_{0}\left(\sigma^{q_{\lambda}}\right)$ is a subgroup, there exists a projection $e_{\lambda} \in Z\left(M^{\sigma}\right)$ such that $0<e_{\lambda} \leq q_{\lambda}$ and $\operatorname{sp} \sigma^{e_{\lambda}} \cap I_{\delta}(\lambda)=\emptyset$ for some $\delta>0$. Here we may assume that the above $\varepsilon$ is less than $\delta / 2$. Since $\bar{e}_{\lambda} \leq \bar{e}$, it follows from Lemma 3.1 that there are projections $e_{1}$ and $e_{2}$ in $Z\left(M^{\sigma}\right)$ such that $e_{1} \leq e_{\lambda}, 0<e_{2} \leq e$ and $\operatorname{sp} \sigma^{e_{2}} \subset \operatorname{sp} \sigma^{e_{1}}+I_{\delta / 2}(0)$. This inclusion relation contradicts with the fact that

$$
I_{\delta / 2}(\lambda) \cap \operatorname{sp} \sigma^{e_{2}} \neq \emptyset, I_{\delta / 2}(\lambda) \cap\left(\operatorname{sp} \sigma^{e_{1}}+I_{\delta / 2}(0)\right)=\emptyset .
$$

Thus $\Gamma_{0}\left(\sigma^{q_{\lambda}}\right)=\lambda \boldsymbol{Z}$ and hence (v) follows.

Remark 4.1. Let $G$ be the additive group $\boldsymbol{R}$ and $\sigma \in \operatorname{Rep}(\boldsymbol{R}, M)$. If $\operatorname{sp} \sigma$ is compact, then $\Gamma_{0}(\sigma)=\Gamma_{1}(\sigma)=\{0\}$.

From the above proposition we have the following one.

Proposition 4.2. The condition (B) implies the condition (C), if one of the following two assumptions is satisfied:

(i) $G=G(\sigma)$; and

(ii) $G$ is the additive group $\boldsymbol{R}$ or $\boldsymbol{Z}$ with the usual topology, $G(\sigma) \neq\{0\}$ and $\Gamma_{0}(\sigma) \neq\{0\}$.

For any $\sigma$ and $\sigma^{\prime}$ in $\operatorname{Rep}(G, M), \sigma \sim \sigma^{\prime}$ if there exists a strongly continuous mapping $u$ of $G$ to the unitaries in $M$ such that $u_{s+t}=u_{s} \sigma_{s}\left(u_{t}\right)$ and $\sigma_{t}{ }^{\prime}(x)=u_{t} \sigma_{t}(x) u_{t}{ }^{*}$ for $s, t \in G$ and $x \in M$. This equivalence relation 
" $"$ is called an "exterior equivalence" by Connes. The following lemma follows immediately from Lemma 3.1.

Lemma 4.3. If $\sigma \sim \sigma^{\prime}$, then $\Gamma_{1}(\sigma)=\Gamma_{1}\left(\sigma^{\prime}\right)$.

The following lemma is used to relate the $\Gamma_{0}(\sigma)$ with the algebraic invariant $\mathrm{S}(M)$ which was defined in Section 1 for a general von Neumann algebra $M$.

Lemma 4.4. Assume either that $G(\sigma)$ is discrete or that $G(\sigma)$ is closed and satisfies the second axiom of countability and $M_{*}$ is separable. Then there exists a $\sigma^{\prime} \in \operatorname{Rep}(G, M)$ such that $\sigma^{\prime} \sim \sigma, M^{\sigma} \subset M^{\sigma^{\prime}}$, $Z\left(M^{\sigma^{\prime}}\right) \subset Z\left(M^{\sigma}\right)$ and $G(\sigma)=G\left(\sigma^{\prime}\right)=\left(\operatorname{sp} \sigma^{\prime}\right)^{\perp}$.

Proof. By similar discussions as in the proof of Lemma 3.4 and Remark 3.1, we have a strongly continuous unitary representation $v$ of $G(\sigma)$ in $Z\left(M^{\sigma}\right)$ such that $\sigma_{s}(x)=v_{s} x v_{s}^{*}$ for $x \in M$ and $s \in G(\sigma)$. Since $G(\sigma)$ is a closed subgroup of $G$, it follows from [6, Lemma 3.3.12] that there exists a strongly continuous unitary representation $u$ of $G$ in $Z\left(M^{\sigma}\right)$ such that $u_{s}=v_{s}$ for $s \in G(\sigma)$. Define a $\sigma^{\prime} \in \operatorname{Rep}(G, M)$ by $\sigma_{t}{ }^{\prime}(x) \equiv u_{t}{ }^{*} \sigma_{t}$ $(x) u_{t}$ for $t \in G$ and $x \in M$. Since $u_{t+s}=\sigma_{t}\left(u_{s}\right) u_{t}$, we have $\sigma^{\prime} \sim \sigma$ and

$$
G(\sigma)=\left\{t \in G: \sigma_{t}^{\prime}=1\right\} \subset G\left(\sigma^{\prime}\right) .
$$

Since $u_{t} \in M^{\sigma} \subset M^{\sigma^{\prime}}$, if $y \in Z\left(M^{\sigma^{\prime}}\right)$, then $\sigma_{t}(y)=u_{t} \sigma_{t}{ }^{\prime}(y) u_{t}{ }^{*}=y$ and hence $y \in M^{\sigma}$. Since $M^{\sigma} \subset M^{\sigma^{\prime}}, y \in\left(M^{\sigma^{\prime}}\right)^{\prime} \subset\left(M^{\sigma}\right)^{\prime}$ and hence $y \in Z\left(M^{\sigma}\right)$. Thus $Z\left(M^{\sigma^{\prime}}\right) \subset Z\left(M^{\sigma}\right)$. If $\sigma_{s}{ }^{\prime}(x)=w x w^{*}$ for $s \in G\left(\sigma^{\prime}\right)$ and a unitary $w \in Z$ $\left(M^{\sigma^{\prime}}\right)$, then $u_{s} w \in Z\left(M^{\sigma}\right)$ and hence $G\left(\sigma^{\prime}\right) \subset G(\sigma)$. Thus $G(\sigma)=G\left(\sigma^{\prime}\right)$.

Since $G\left(\sigma^{\prime}\right)=\left(\operatorname{sp} \sigma^{\prime}\right)^{\perp}$ is clear, we complete the proof.

Proof of Proposition 4.2. (i) If $G\left(\sigma^{e}\right)=G$, then $\Gamma_{0}(\sigma) \subset \Gamma_{1}(\sigma)$ $\subset\{0\}$ by Lemma 2.6 , which implies the condition (C).

(ii) By (i) it suffices to consider the case $G(\sigma) \neq G$.

The case where $G(\sigma)=(\operatorname{sp} \sigma)^{\perp}$. Suppose that $G=\boldsymbol{R}$ (resp. $\boldsymbol{Z}$ ). Since $\Xi \equiv G(\sigma)^{\perp}$ is discrete and $G(\sigma) \neq G$ by assumption, there exists a generator $\gamma \in \boldsymbol{R}$ (resp. $[0,2 \pi)$ ) of $\Xi$. Here we apply Proposition 4.1 to 
$\sigma$. Since $G(\sigma) \neq\{0\}$ by assumption, $q_{\infty}=1$. Since $\Gamma_{0}(\sigma) \neq\{0\}$ by assumption, we have $q_{0}=0$. Since $\Xi$ is discrete, we have a partition $\left\{p_{n}\right.$ : $n \in \mathbf{N}\}$ (resp. $\left\{p_{n}: n \mid m\right\}, m \equiv 2 \pi / \gamma$ ) in $Z\left(M^{\sigma}\right) \cap Z(M)$ of the identity such that

$$
\Gamma_{0}\left(\sigma^{p_{n}}\right)=\Gamma_{1}\left(\sigma^{p_{n}}\right)=n \gamma Z(\operatorname{resp} .\{n \gamma: n \mid m\}),
$$

which is the condition (C) for $\sigma^{p_{n}}$ over $M_{p_{n}}$. Since $\widehat{G} / n \gamma Z$ is compact, the conditions $\left(\mathrm{B}^{\prime}\right)$ and (C) are equivalent over $M_{p_{n}}$ by (ii) of Theorem 1.1 and hence $G\left(\sigma^{e}\right)^{\perp}=n \gamma \boldsymbol{Z}$ (resp. $\{n \gamma: n \mid m\}$ ) for all non zero $e \in Z\left(M^{\sigma}\right.$ ) with $e \leq p_{n}$. On the other hand, the condition (B) implies $G\left(\sigma^{e}\right)^{\perp}=\Xi$ for all $e \in Z\left(M^{\sigma}\right)$. Thus $p_{n}=1$ and $p_{m}=0(m \neq n)$ for some $n \in \boldsymbol{N}$, namely, $\Xi=n \gamma \boldsymbol{Z}$. Since $\gamma$ is a generator of $\Xi, n=1$. Thus $\Gamma_{0}(\sigma)=\Gamma_{1}(\sigma)=\Xi$.

The general case. For a given $\sigma$ we choose a $\sigma^{\prime}$ as in Lemma 4.4. Then $G(\sigma)=G\left(\sigma^{\prime}\right)=\left(\operatorname{sp} \sigma^{\prime}\right)^{\perp}$ and hence $\Gamma_{0}\left(\sigma^{\prime}\right)=\Gamma_{1}\left(\sigma^{\prime}\right)=G\left(\sigma^{\prime}\right)^{\perp}$ from the above. Since $G\left(\sigma^{\prime}\right)=G(\sigma)=\Xi^{\perp}$ by the condition (B), we have $\Gamma_{0}\left(\sigma^{\prime}\right)=\Gamma_{1}\left(\sigma^{\prime}\right)=\Xi$. Therefore $\Gamma_{1}\left(\left(\sigma^{\prime}\right)^{e}\right)=\Xi$ for all non zero $e \in Z\left(M^{\sigma}\right)$. Since $M^{\sigma} \subset M^{\sigma^{\prime}}$ by Lemma 4.4, if $e$ is a projection in $Z\left(M^{\sigma}\right), \Gamma_{1}\left(\sigma^{e}\right)$ $=\Gamma_{1}\left(\left(\sigma^{\prime}\right)^{e}\right)$ by Lemma 4.3 , and hence $\Gamma_{0}(\sigma)=\Gamma_{1}(\sigma)=\Xi$, which is the condition (C).

Q.E.D.

Proof of Theorem 1.2. Since $\widehat{G} / \Gamma_{0}\left(\sigma^{\phi}\right)$ is compact and $\Gamma_{0}\left(\sigma^{\phi}\right)$ $=\Gamma_{1}\left(\sigma^{\phi}\right)$ by assumption, the condition $\left(\mathrm{B}^{\prime}\right)$ holds by (ii) of Theorem 1.1 and hence $G(\sigma)=\Gamma_{0}\left(\sigma^{\phi}\right)^{\perp}$ is discrete. Applying Lemma 4.4, we have a $\sigma \in \operatorname{Rep}(\boldsymbol{R}, M)$ such that $\sigma \sim \sigma^{\phi}$ and $\operatorname{sp} \sigma \subset G\left(\sigma^{\phi}\right)^{\perp}$. The condition $\left(\mathrm{B}^{\prime}\right)$ implies that

$$
G\left(\sigma^{\phi}\right)=G\left(\left(\sigma^{\phi}\right)^{e}\right)=\Gamma_{0}\left(\sigma^{\phi}\right)^{\perp} \quad \text { and } \quad \Gamma_{0}\left(\sigma^{\phi}\right) \subset \operatorname{sp}\left(\sigma^{\phi}\right)^{e}
$$

for all non zero $e$ in $Z\left(M^{\sigma^{\phi}}\right)$ and hence that

$$
\operatorname{sp} \sigma \subset G\left(\sigma^{\phi}\right)^{\perp}=I_{0}\left(\sigma^{\phi}\right) \subset \operatorname{sp}\left(\sigma^{\phi}\right)^{e} .
$$

Since $\sigma \sim \sigma^{\phi}$ implies $\sigma=\sigma^{\psi}$ for some $\psi \in W(M)$ by [6, Theorem 1.2.4], we have

$$
\bigcap\left\{\operatorname{sp} \sigma^{\psi}: \psi \in W(M)\right\} \subset \Gamma_{0}\left(\sigma^{\phi}\right) .
$$

On the other hand, since $\sigma^{\phi} \sim \sigma^{\psi}$ for all $\psi$ in $W(M)$, it follows from Lemma 4.3 that 


$$
\Gamma_{0}\left(\sigma^{\phi}\right) \subset \Gamma_{1}\left(\sigma^{\phi}\right)=\Gamma_{1}\left(\sigma^{\psi}\right) \subset \operatorname{sp} \sigma^{\psi}
$$

and hence

$$
\Gamma_{0}\left(\sigma^{\phi}\right)=\bigcap\left\{\operatorname{sp} \sigma^{\psi}: \psi \in W(M)\right\} .
$$

Since sp $\sigma^{\psi}=\log \left(\operatorname{Sp}\left(A_{\psi}\right) \cap \boldsymbol{R}_{+}{ }^{*}\right)$ by $\left[6\right.$, Lemma 3.2.2] and $Z(M) \subset M \sigma^{\sigma^{\psi}}$ for $\psi \in W(M)$, we have a desired result.

\section{§ 5. Unbounded Derivation}

Before going into the definition of a derivation, we recall that $\sigma(g)$ is $\sigma$-weakly continuous on $M$ for $g \in L^{1}(G)$. For the sake of completeness we shall give a slightly different proof from [2, Proposition 1.4].

Let $M_{1}$ be the unit ball of $M$ with the $\sigma$-weak topology. Choose a compact $K \subset G$ for a given $\varepsilon>0$ such that

$$
\int_{G \backslash \mathbf{K}}|g(t)| d t<\varepsilon
$$

Since the dual representation $\sigma^{\prime}$ on $M_{*}$ of $\sigma \in \operatorname{Rep}(G, M)$ is strongly continuous $[1$, Proposition 1 in $\S 6]$, for any $t_{j} \in G, \varepsilon>0$ and $\phi \in M_{*}$ there exists a neighbourhood $V_{j}$ of $t_{j}$ such that

$$
\sup _{t \in V_{j}} \sup _{x \in M_{1}}\left|\left\langle\left(\sigma_{t}-\sigma_{t_{j}}\right)(x), \phi\right\rangle\right|<2^{-1} \varepsilon
$$

Since $K$ is compact, we can find a finite covering $V_{j}, j=1, \cdots, n$ of $K$. Since $\sigma_{t_{j}}$ is $\sigma$-weakly continuous, there exists a neighbourhood $N_{j}$ of 0 in $M_{1}$ such that $\left|\left\langle\sigma_{t_{j}}\left(N_{j}\right), \phi\right\rangle\right|<2^{-1} \varepsilon$. Set $N \equiv \bigcap_{j=1}^{n} N_{j}$. Since $t \in K$ belongs to some $V_{j}$,

$$
\left|\left\langle\sigma_{t}(x), \phi\right\rangle\right| \leq\left|\left\langle\left(\sigma_{t}-\sigma_{t_{j}}\right)(x), \phi\right\rangle\right|+\left|\left\langle\sigma_{t_{j}}(x), \phi\right\rangle\right|\langle\varepsilon
$$

for all $x \in N$. Therefore

$$
\begin{aligned}
|\langle\sigma(g) x, \phi\rangle| & \leq \varepsilon \int_{K}|g(t)| d t+2\|\phi\| \int_{G \backslash K}|g(t)| d t \\
& \leq\left(\|g\|_{1}+2\|\phi\|\right) \varepsilon
\end{aligned}
$$

for all $x \in N$. Thus $\phi \circ \sigma(g)$ is $\sigma$-weakly continuous on $M_{1}$ and hence on $M$ by Banach's theorem. Consequently, $\sigma(g)$ is $\sigma$-weakly continuous.

Now we shall generalize the concept of a derivation of $M$ to the unbounded case as the following, [7]. 
Definition 5.1. A linear operator $\delta$ on $M$ is called a self-adjoint derivation of $M$ if the domain $\mathrm{D}(\delta)$ of $\delta$ is a $\sigma$-weakly dense *-subalgebra of $M$ and

$$
\delta(x y)=\delta(x) y+x \delta(y), \delta\left(x^{*}\right)=-\delta(x)^{*}
$$

for all $x, y \in \mathrm{D}(\delta)$. In addition, $\delta$ is said to be spatial (resp. inner) if there exists a self-adjoint operator $h$ (resp. $h_{\eta} M$ ) whose domain is invariant under $\mathrm{D}(\delta)$ and which satisfies

$$
\delta(x)=\overline{h x-x h} \equiv \overline{[h, x]}
$$

for all $x \in \mathrm{D}(\delta)$.

For a linear operator $\delta$ on a Banach space $E$, an $x \in E$ is analytic (resp. entire) for $\delta$ if the function $t \in \boldsymbol{R} \mapsto \sum_{n=0}^{\infty}(n !)^{-1} t^{n} \delta^{n} x \in E$ exists and is analytic in some neighbourhood of 0 (resp. entire). For a representation $\sigma$ of $\boldsymbol{R}$ on $E$, an $x \in E$ is analytic (resp. entire) for $\sigma$ if the function $t \mapsto \sigma_{t}(x)$ is analytic in some neighbourhood of 0 (resp. entire).

If $\sigma_{t}, t \in \boldsymbol{R}$ is a strongly continuous one parameter group of uniformly bounded operators on $E$, then

$$
x_{\lambda} \equiv\left(\frac{1}{2 \pi \lambda^{2}}\right)^{1 / 2} \int_{\boldsymbol{R}} \sigma_{t}(x) \exp \left(-\frac{t^{2}}{2 \lambda^{2}}\right) d t
$$

for $x \in E$, are entire for $\sigma$ and $x$ is the limit of $x_{\lambda}$ as $\lambda \rightarrow 0$. Furthermore if $\delta$ is the generator of $\sigma$, then

$$
\sum_{n=0}^{\infty} \frac{t^{n}}{n !}\left\|\delta^{n}\left(x_{\lambda}\right)\right\|<+\infty, \text { for all } t \in \boldsymbol{R}
$$

In the following a linear operator $\delta$ on $M$ is said to be $\sigma$-rveakly closed if the graph of $\delta$ in $M \oplus M$ is $\sigma$-weakly closed.

Proposition 5.1. Let $\sigma \in \operatorname{Rep}(\boldsymbol{R}, M)$ and $\delta$ be a linear operator on $M$ whose domain $\mathrm{D}(\delta)$ is the set of $x \in M$ for which $t^{-1}\left(\sigma_{t}(x)-x\right)$ is $\sigma$-weakly convergent as $t \downarrow 0$, and

$$
\delta x=\lim _{t \downarrow 0}(i t)^{-1}\left(\sigma_{t}(x)-x\right)
$$

for all $x \in \mathrm{D}(\delta)$. Then 
(i) $\mathrm{D}(\delta)$ is a $\sigma$-weakly dense ${ }^{*}$-subalgebra of $M$ and $\delta$ is a selfadjoint $\sigma$-weakly closed derivation of $M$;

(ii) for any non-zero real number $\lambda, \lambda-i \delta$ has the $\sigma$-weakly continuous inverse $(\lambda-i \delta)^{-1}$ and $\left\|(\lambda-i \delta)^{-1}\right\| \leq|\lambda|^{-1}$;

(iii) the set of entire elements for $\delta$ is $\sigma$-reakly dense in $M$;

(iv) $\delta$ is spatial(resp. inner) if and only if $\sigma$ is spatial(resp. inner); and

(v) the infinitesimal generator of the dual representation $\sigma^{\prime}$ of $\sigma$ is the dual of $\delta$.

Conversely, if $\delta$ is a self-adjoint $\sigma$-weakly closed derivation of $M$ and if for any non-zero real number $\lambda, \lambda-i \delta$ has an inverse and $\left\|(\lambda-i \delta)^{-1}\right\| \leq|\lambda|^{-1}$, then there exists a unique representation $\sigma \in \operatorname{Rep}(\boldsymbol{R}$, $M)$ of which $\delta$ is an infinitesimal generator.

Proof. (i, ii) It is clear that $\mathrm{D}(\delta)$ is a *-subalgebra of $M$ and $\delta$ is a self-adjoint derivation of $M$. Define $\phi_{\lambda}$ for $\lambda>0$ by

$$
\phi_{\lambda}=\int_{0}^{\infty} \lambda \sigma_{t} \exp (-\lambda t) d t
$$

Applying the same argument as the one parameter semi-group theory on a Banach space, we know that the range of $\phi_{\lambda}$ coincides with $\mathrm{D}(\delta)$, that $\lambda^{-1} \phi_{\lambda}=(\lambda-i \delta)^{-1}$ and that $\phi_{\lambda}(x)$ converges $\sigma$-weakly to $x$ as $\lambda \rightarrow \infty$ for $x \in M$. Therefore $\mathrm{D}(\delta)$ is $\sigma$-weakly dense in $M$. Since $\phi_{\lambda}$ is $\sigma$-weakly continuous as shown at the begining of this Section, $\delta$ is $\sigma$-weakly closed.

(iii) $x_{\lambda}$ in $(5 \cdot 1)$ is entire for $\delta$ and $\sigma$-weakly converges to $x$ as $\lambda \rightarrow \infty$. Therefore we conclude (iii).

(iv) Suppose that $\sigma$ is spatial(resp. inner). There exists a self-adjoint operator $h$ (resp. $h \eta M)$ such that $\sigma_{t}(x)=u_{t} x u_{t}{ }^{*}$ and $u_{t}=\exp (i t h)$. Since

$$
(i t)^{-1}\left(\sigma_{t}(x)-x\right) \xi=u_{t} x(i t)^{-1}\left(u_{t}^{*}-1\right) \xi+(i t)^{-1}\left(u_{t}-1\right) x \xi,
$$

if $x \in \mathrm{D}(\delta)$ and $\xi$ is in the domain $\mathrm{D}(h)$ of $h$, then $x \xi \in \mathrm{D}(h)$ and $\delta(x) \xi=[h, x] \xi$. Since $\mathrm{D}(h)$ is dense in $\mathcal{H}$, we have $\delta x=\overline{[h, x]}$.

Conversely, suppose that $\delta$ is spatial(resp.inner). Let $h$ be a selfadjoint operator which induces $\delta$ as in Definition 5.1. Put $u_{t} \equiv \exp (i t h)$. Denote by $\mathcal{H}^{(e)}\left(\operatorname{resp} . M^{(e)}\right)$ the set of entire elements for $u($ resp. $\delta)$. 
We shall show by induction that $x \mathcal{G}^{(e)} \subset \mathrm{D}\left(h^{n}\right)$ for $n \in \mathbb{N}$ and $x \in M^{(e)}$. By the assumption for $h, x \mathcal{F}^{(e)} \subset \mathrm{D}(h)$. If $x \mathcal{H}^{(e)} \subset \mathrm{D}\left(h^{n}\right)$, then

$$
\delta^{n} x \xi=\sum_{k=0}^{n}\left(\begin{array}{l}
n \\
k
\end{array}\right) h^{k} x(-h)^{n-k} \xi,
$$

for $\xi \in \mathcal{F}^{(e)}$. Since $\left(\delta^{n} x\right) \xi \in \mathrm{D}(h)$, we know that

$$
h^{n} x \xi=\left(\delta^{n} x\right) \xi-\sum_{k=0}^{n-1}\left(\begin{array}{l}
n \\
k
\end{array}\right) h^{k} x(-h)^{n-k} \xi
$$

is in $\mathrm{D}(h)$ and hence $x \xi \in \mathrm{D}\left(h^{n+1}\right)$.

If $x \in M^{(e)}$ and $\xi, \eta \in \mathcal{H}^{(e)}$, then

$$
\begin{aligned}
\left(\sigma_{t}(x) \xi \mid \eta\right) & =\sum_{n=0}^{\infty}(n !)^{-1}(i t)^{n}\left(\left(\delta^{n} x\right) \xi \mid \eta\right) \\
& =\sum_{n=0}^{\infty} \frac{(i t)^{n}}{n !}\left(\sum_{k=0}^{n}\left(\begin{array}{l}
n \\
k
\end{array}\right) h^{k} x(-h)^{n-k} \xi \mid \eta\right) \\
& =\sum_{n=0}^{\infty} \sum_{k=0}^{n}\left(x \frac{(i t)^{n-k}}{(n-k) !}(-h)^{n-k} \xi \mid \frac{(i t)^{k}}{k !}(-h)^{k} \eta\right) .
\end{aligned}
$$

Since $\xi, \eta \in \mathcal{H}^{(e)}$, the right hand side is absolutely convergent. Therefore

$$
\begin{aligned}
\left(\sigma_{t}(x) \xi \mid \eta\right) & =\left(x \sum_{n=0}^{\infty}(n !)^{-1}(-i t h)^{n} \xi \mid \sum_{m=0}^{\infty}(m !)^{-1}(-i t h)^{m} \eta\right) \\
& =(x(\exp (-i t h)) \xi \mid \exp (-i t h) \eta) \\
& =((\exp (i t h)) x(\exp (-i t h)) \xi \mid \eta) .
\end{aligned}
$$

Since $\mathcal{H}^{(e)}$ is dense in $\mathscr{H}$ and $M^{(e)}$ is $\sigma$-weakly dense in $M$, we have $\sigma_{t}(x)=(\exp (i t h)) x(\exp (-i t h))$ for $x \in M$.

(v) Let $\delta^{\prime}$ and ${ }^{t} \delta$ be the infinitesimal generator of the dual $\sigma^{\prime}$ on $M_{*}$ of $\sigma$ and the dual of $\delta$, respectively. For $\lambda>0$, the dual of $(\lambda-i \delta)^{-1}$ is $\left(\lambda-i^{t} \delta\right)^{-1}$. Since $\delta^{\prime} \subset{ }^{t} \delta,\left(\lambda-i \delta^{\prime}\right)^{-1} \subset\left(\lambda-i^{t} \delta\right)^{-1}$. Since the domain of $\left(\lambda-i \delta^{\prime}\right)^{-1}$ is $M_{*}$, we have $\left(\lambda-i \delta^{\prime}\right)^{-1}=\left(\lambda-i^{t} \delta\right)^{-1}$ and hence, $\delta^{\prime}={ }^{t} \delta$.

Suppose that $\delta$ is a self-adjoint $\sigma$-weakly closed derivation of $M$ and that $\left\|(\lambda-i \delta)^{-1}\right\| \leq|\lambda|^{-1}$ for any $\lambda \neq 0$. Denote by $\delta^{\prime}$ the dual of $\delta$ on $M_{*}$. Since $\left\|\left(\lambda-i \delta^{\prime}\right)^{-1}\right\| \leq|\lambda|^{-1}$, by the Hille-Yosida theorem, $\delta^{\prime}$ is the generator of a strongly continuous contraction one parameter group $\sigma^{\prime}$ on $M_{*}$. The dual $\sigma$ of $\sigma^{\prime}$ is a $\sigma$-weakly continuous contraction one parameter group on $M$. Moreover (v) is valid for $\sigma$ and the generator of $\sigma$ is 
$\sigma$-weakly closed. Since the bidual of a closed linear map is itself, the generator of $\sigma$ is the dual of $\delta^{\prime}$, namely, $\delta$. Therefore we have for any entire elements $x$ and $y$,

$$
\begin{gathered}
\sigma_{t}(x) \sigma_{t}(y)=\sum_{n=0}^{\infty} \frac{(i t)^{n}}{n !} \delta^{n}(x) \sum_{n=0}^{\infty} \frac{(i t)^{n}}{n !} \delta^{n}(y)=\sum_{n=0}^{\infty} \frac{(i t)^{n}}{n !} \delta^{n}(x y)=\sigma_{t}(x y), \\
\sigma_{t}\left(x^{*}\right)=\sum_{n=0}^{\infty} \frac{(i t)^{n}}{n !} \delta^{n}\left(x^{*}\right)=\sum_{n=0}^{\infty} \frac{(-i t)^{n}}{n !} \delta^{n}(x)^{*}=\sigma_{t}(x)^{*} .
\end{gathered}
$$

Since (iii) is valid for $\delta$, we conclude the multiplicativity and self-adjointness of $\sigma_{t}$. Therefore $\sigma_{t}$ is a ${ }^{*}$-automorphism.

Q.E.D.

Remark 5.1. In the above proposition $M^{(e)}$ is a core of $\delta$ with respect to the $\sigma$-weak topology on $M$. Indeed, if $x \in \mathrm{D}(\delta)$, then $x_{\lambda}$ defined by $(5 \cdot 1)$ converges $\sigma$-weakly to $x$. Furthermore $\delta\left(x_{\lambda}\right)=(\delta x)_{\lambda}$ converges $\sigma$-weakly to $\delta x$ as $\lambda \rightarrow 0$.

Lemma 5.1. If $\delta$ is the infinitesimal generator of $\sigma \in \operatorname{Rep}(\boldsymbol{R}, M)$, then $\operatorname{Sp} \delta=\operatorname{sp} \sigma$.

Proof. Suppose that $\lambda \in \operatorname{sp} \sigma=-\operatorname{sp} \sigma$ and $\langle t, \lambda\rangle \equiv \exp (i t \lambda)$ for $t \in \boldsymbol{R}$. Define a function $g \in \mathrm{L}^{1}(\boldsymbol{R})$ for any $\alpha>0$ by

$$
g(t) \equiv \exp (-\alpha t) \overline{\langle t, \lambda\rangle} \quad(t>0) ; g(t) \equiv 0 \quad(t \leq 0) .
$$

Since $i(\delta-\lambda)$ is the infinitesimal generator of a one parameter group $t \mapsto \overline{\langle t, \lambda\rangle} \sigma_{t}$, we have

$$
\sigma(g)=\int_{0}^{\infty} \exp (-\alpha t) \overline{\langle t, \lambda\rangle} \sigma_{t} d t=-i(\lambda-i \alpha-\delta)^{-1}
$$

and $\hat{g}(-\lambda)=\alpha^{-1}$. Therefore, by $[6$, Lemma 2.3.6], we have $\|(\lambda-i \alpha$ $-\delta)^{-1} \| \geq \alpha^{-1}$ and hence

$$
\lim _{\alpha \downarrow 0}\left\|(\lambda-i \alpha-\delta)^{-1}\right\|=\infty .
$$

Consequently, $\lambda \in \operatorname{Sp} \delta$.

Assume that

$$
\lim _{\alpha \downarrow 0}\left\|(\lambda-i \alpha-\delta)^{-1}\right\|<\infty
$$

By the resolvent equation, $(\lambda-i \alpha-\delta)^{-1}$ converges in norm to a bounded 
operator $\rho$ as $\alpha \downarrow 0$ and $\rho=(\lambda-\delta)^{-1}$. Therefore $\lambda \in \operatorname{Sp} \delta$ implies (5.3) and hence that there exist for any $\varepsilon>0$ a positive $\alpha \in \boldsymbol{R}$ and a non zero $y \in M$ such that $2 \alpha<\varepsilon$ and

$$
\varepsilon\left\|(\lambda-i \alpha-\delta)^{-1} y\right\|>2\|y\| .
$$

By putting $x \equiv\|z\|^{-1} z$ for $z \equiv(\lambda-i \alpha-\delta)^{-1} y$, we have

$$
\|(\lambda-\delta) x\| \leq\|(\lambda-i \alpha-\delta) x\|+\|i \alpha x\|<\varepsilon .
$$

From the equation

$$
\overline{\langle t, x\rangle} \sigma_{t}(x)-x=\int_{0}^{t} \overline{\langle s, \lambda\rangle} \sigma_{s} \circ(i(\delta-\lambda))(x) d s,
$$

it follows that

$$
\left\|\sigma_{t}(x)-\langle t, \lambda\rangle x\right\|<|t| \varepsilon .
$$

Therefore, by $[6$, Lemma 2.3.6], we have $-\lambda \in \operatorname{sp} \sigma=-\operatorname{sp} \sigma$. Q.E.D.

Lemma 5.1 and Theorem 1.1 give following corollaries. It is clear that $x \in M^{\sigma}$ if and only if $\delta x=0$. Therefore the restriction $\delta^{e}$ of $\delta$ to $M_{e}$ is a derivation corresponding to $\sigma^{e}$. For a derivation $\delta$, we denote by $M^{\delta}$ the set $\{x \in M: \delta x=0\}$.

Corollary 5.1. Let $\delta$ be a derivation of $M$ which is the infinitesimal generator of a representation in $\operatorname{Rep}(\boldsymbol{R}, M)$. The following conditions are equivalent for $\lambda>0$ :

(i) $\cap\left\{\mathrm{Sp} \delta^{e}: e \in M^{\delta}, e \neq 0\right\}=\bigcap\left\{\operatorname{Sp} \delta^{e}: e \in M^{\delta}, \bar{e}=1\right\}=\lambda Z$; and

(ii) for any non zero projection $f$ in $Z\left(M^{\delta}\right)$ and for any neighbourhood $V$ of 0 , there exists a non zero projection $e$ in $Z\left(M^{\delta}\right)$ such that $e \leq f$ and $\lambda Z \in \operatorname{Sp} \delta^{e} \subset \lambda Z+V$.

Corollary 5.2. Let $\delta$ be a derivation of $M$ which is the infinitesimal generator of a representation in $\operatorname{Rep}(\boldsymbol{R}, M)$. If $M_{*}$ is separable, then the following conditions are equivalent:

(i) $\delta$ is inner; and

(ii) for any non zero projection $f$ in $Z\left(M^{\delta}\right)$ and for any $\varepsilon>0$ there exists a non zero projection $e$ in $Z\left(M^{\delta}\right)$ such that $e \leq f$ and $\left\|\delta^{e}\right\| \leq \varepsilon$. 
Since the separability of $M_{*}$ is unnecessary for the implication (ii) $\Rightarrow$ (i) in Corollary 5.2, we have Corollary 5.3, which is a restatement of a result of Borchers [3, Theorem]. We shall restate it more precisely.

Corollary 5.3. Let $\delta$ be a derivation of $M$ which is the infinitesimal generator of a representation in $\operatorname{Rep}(\boldsymbol{R}, M)$. If there is a non negative self-adjoint operator $k$ implementing $\delta$, then $\delta$ is inner, and a self-adjoint operator $h \eta M$ implementing $\delta$ is uniquely determined by the condition that $2\|h e\|=\left\|\delta^{e}\right\|$ for all $e \in Z\left(M^{\delta}\right)$. In particular, Sp $(h e)+\|h e\| \subset \operatorname{Sp} \delta^{e} \cap \boldsymbol{R}_{+}$.

\section{Acknowledgements}

The authors would like to thank Dr. O. Bratteli for pointing out an error on a derivation and Professor $\mathrm{H}$. Araki for taking pains of reading the manuscript carefully and indicating some errors.

\section{References}

[1] Aarnes, J. F., Continuity of group representations, with applications to $C^{*}$-algebras, J. Functional Analysis, 5 (1970), 14-36.

[2] Arveson, W., On groups of automorphisms of operator algebras, J. Functional Analysis, 15 (1974), 217-243.

[3] Borchers, H. J., Energy and momentum as observables in quantum field theory, Comm. Math. Phys., 2 (1966), 49-54.

[4] Characterization of inner *automorphisms of $W^{*}$-algebras, Publ. RIMS, Kyoto Univ., 10 (1974), 11-49.

[5] Choda, H., On a decomposition of automorphisms of von Neumann algebras, Proc. Japan Acad., 49 (1973), 809-811.

[6] Connes, A., Une classification des facteurs de type III, Ann. Sci. École Norm. Sup., 6 (1973), 133-252.

[7] Gille, J. F., An exponentiation theorem for unbounded derivations, Ann. Inst. H. Poincaré, 13 (1970), 215-220.

[8] Ikunishi, A. and Nakagami, Y., Automorphism group of von Neumann algebras and semi-finiteness of an infinite tensor product of von Neumann algebras, to appear.

[9] - On an invariant $\Gamma$ for an automorphism group of a von Neumann algebra, Japan-U. S. Seminar on $C^{*}$-algebras and Applications to Physics, (1974), 185-189.

[10] Kadison, R. and Ringrose, J. R., Derivations and automorphisms of operator algebras, Comm. Math. Phys., 4 (1967), 32-64.

[11] Kallman, R. R., Groups of inner automorphisms of von Neumann algebras, $J$. Functional Analysis, 7 (1971), 43-60. 
[12] Moore, C. C., Restrictions of unitary representations to subgroups and ergodic theory: group extensions and group cohomology, in Group representations in Mathematics and Physics, edited by V. Bergmann, Lecture Notes in Phys. 6, pp. 1-35, Springer-Verlag, Berlin, 1969.

[13] Parthasarathy, K. R., Multipliers on locally compact groups, Lecture Notes in Math. 93, Springer-Verlag, Berlin, 1969.

[14] Sakai, S., $C^{*}$-algebras and $W^{*}$-algebras, Springer-Verlag, Berlin, 1971. 